\title{
miRNA-124-3p.1 Inhibits the Osteogenic and Odontogenic Differentiation of SCAPs via MACF1/smad7 Axis
}

$\mathrm{Na} \mathrm{Li}$

Nanjing Medical University

Zehan Li

Nanjing Medical University

Ming Yan

Nanjing Medical University

Yanqiu Wang

Nanjing Medical University

Yongchun Gu

Nanjing Medical University

Jintao Wu

Nanjing Medical University

Chaoting Yan

Nanjing Medical University

Tong Xiao

Nanjing Medical University

Lin Fu

Nanjing Medical University

Jinhua Yu ( $\nabla$ yujinhua@njmu.edu.cn )

Nanjing Medical University https://orcid.org/0000-0003-4874-9910

\section{Research Article}

Keywords: miRNA-124-3p.1, osteogenetic and odontogenetic differentiation, stem cells from apical papillae, MACF1

Posted Date: December 13th, 2021

DOI: https://doi.org/10.21203/rs.3.rs-1121374/v1

License: (c) (i) This work is licensed under a Creative Commons Attribution 4.0 International License. Read Full License 


\section{Abstract}

Background: Previous research has indicated that altered expression of micro-RNAs (miRNAs) is in connection with differentiation of stem cells from apical papillae (SCAPs). We investigated the mechanisms that miR-124-3p.1 inhibited osteogenic and odontogenic differentiation of SCAPs.

Methods: SCAPs were isolated from dental apical papilla. MiR-124-3p.1 mimic and inhibitor were used for overexpression and knockdown assays. For overexpression and knockdown of microtubule actin crosslinking factor 1 (MACF1), lentivirus infection and siRNA transfection were performed. Luciferase reporter assay was performed to determine the relationship between miR-124-3p.1 and MACF1. The osteogenic and odontogenic differentiation potential was analyzed by alkaline phosphatase activity analysis (ALP), alizarin red S (ARS) staining, quantitative real time reverse-transcription polymerase chain reaction (qRTPCR), western blot and immunofluorescence (IF) staining.

Results: We observed a time dependent decrease of miR-124-3p.1 level in mineralization induction of SCAPs. Further study found that miR-124-3p.1 exhibited an inhibitory effect on SCAPs osteo/odontogenic differentiation. Similarly, we found that the overexpression of miR-124-3p.1 dramatically inhibited MACF1 protein level in SCAPs and knockdown of miR-124-3p.1 significantly increased MACF1 protein level in SCAPs. Moreover, MACF1 was verified as the targeting of miR-124-3p.1. Meanwhile, the expression of MACF1 was related to smad7 nuclear translocation.

Conclusion: Collectively, diverse data demonstrated that miR-124-3p. 1 is a regulator of MACF1/smad7, playing plays an important role in osteogenic and odontogenic differentiation of SCAPs via MACF1/smad7 axis.

\section{Introduction}

Pulpitis and periapical periodontitis are common oral diseases. Root canal treatment (RCT) is commonly used to treat these problems and relieve the pain of patients. But the traditional method is limited by the incomplete root formation with the open apex and short roots[1]. Treating immature permanent teeth with pulpitis and periapical periodontitis is a challenge in dental medicine[2]. The immature pulpless tooth with an open apex become easy to fracture, resulting in a higher incidence of tooth extraction [3]. Adult stem cells play a significant role in maintaining homeostasis of tissue and in the process of tissue repair and regeneration [4, 5]. In fact, adult mesenchymal stem cells (MSCs) are pluripotent adult stem cells that are isolated from a variety of tissues or organs[6]. Additionally, a recent report indicates that MSCs have chondrogenic, osteogenic, or adipogenic differentiation potential. Stem cells from the tooth mesoderm derived from neural crest cells may be sources for tissue regeneration, including stem cells from apical papilla (SCAPs) [7, 8]. SCAPs residing in the apical papilla play a critical role in tooth development and pulp regeneration in permanent teeth[1]. SCAPs were classified as pluripotent MSCs after a positive expression of CD146, CD90, CD73, STRO-1 and CD105 markers[8]. SCAPs t exhibit the properties of high proliferative potential, low immunogenicity, self-renewal capacity and multipotency of differentiation 
such as osteogenetic, odontogenetic, dentinogenetic, adipogenetic and chondrogenic $[9,10]$. In addition, considerable evidence indicates that after culture in mineral induction medium (MM) containing $\beta$ glycerophosphate, dexamethasone, and L-ascorbate-2-phosphate, SCAPs are found to express a variety of osteo/odontogenic markers, such as ALP, osterix (OSX), runt-related transcription factor 2 (RUNX2) and dentin sialophosphoprotein (DSPP) [11]. Previous studies have found DSPP, RUNX2, ALP and OSX to be important transcriptional regulators of osteogenic and odontogenic differentiation[12]. SCAPs were used to reconstruct the spinal cord injury in animal model[13]. Therefore, SCAPs have been increasingly employed to investigate regenerative medicine.

MiRNAs are a class of highly conserved small noncoding RNAs molecules that operates as genome main regulators and fine tuners via post-transcriptional gene silencing[14]. MiRNAs containing 19-22 nucleotides RNA molecules, always act as a negative regulator in the process of target genes expression in a sequence-specific manner by directly binding partially complementary sequences of target mRNAs in 3'UTRs leading to suppression of the expression of their target mRNAs and its corresponding protein in many biological processes $[15,16]$. MiRNAs extensively distributed in the body[17]. Evidences showed that miRNAs are ubiquitous and potent regulators of almost all biological processes, including proliferation, cell differentiation, metabolism, tumorigenesis, apoptosis, and tissue development[18-20]. Emerging evidence also suggests that miRNAs are active regulators in the self-renewal ability and differentiation potential of stem cells by post-transcriptionally targeting factors implicated in stem cell maintenance[12, 21]. It is reported that increasing number of miRNAs exert a significant impact on apoptosis, proliferation, differentiation and bone resorption of osteoblasts[19, 22]. There are studies have demonstrated that miRNAs almost function in the whole process of osteogenic differentiation[23, 24]. Most of these miRNAs have been reported to promote or inhibit the formation and maturation of osteoblasts, and a few miRNAs are involved in the regulation of osteoblasts functions[25]. Recently, some studies have demonstrated the importance of miRNAs in regulating osteogenic differentiation[26]. For instance, miR-138 reduces ectopic bone formation in vivo through negatively regulating osteogenic differentiation of human MSCs[27]. MiR-124 also inhibits osteogenic differentiation of MSCs and in vivo bone formation[28]. Our team have demonstrated that miRNA let7b played the important role in the differentiation of SCAPs[29]. Previous studies reported that miR-124-3p.1 is involved in cancer development and has also been identified as a potential tumor suppressor in certain cancers[30-32]. Moreover, miR-124-3p.1 sensitizes carboplatin induced mitochondrial apoptosis by inhibiting CAV1 in ovarian cancer[33]. Recently, there are studies demonstrated that miR-124-3p.1 overexpression negatively regulated proliferation and osteogenic differentiation of human bone marrow mesenchymal stem cells (BMSCs)[34]. However, the role of miR-124-3p.1 in the differentiation of SCAPs are still unclear. The purpose of this study was to explore the effect and mechanism of miR-124-3p.1 on proliferation and osteo/odontogenic differentiation of SCAPs.

\section{Materials And Methods}

\subsection{Cell culture}


SCAPs were isolated from dental apical papilla tissue. Dental apical papillae were isolated and transferred into phosphate-buffered saline (PBS; Gibco) containing penicillin-streptomycin (Pen-Strep; Gibco) and trimmed to small pieces under sterile conditions. The samples containing stem cells were transferred into a flask containing a-modified Eagle's minimum essential medium (a-MEM; Gibco), 10\% fetal bovine serum (FBS; $\mathrm{BI}$ ) and $2 \%$ Pen-Strep.

\subsection{Characterization of SCAPs}

\subsubsection{Immunofluorescence staining (IF)}

SCAPs were cultured for 3 days. Briefly, cells were incubated with primary antibody STRO-1 (eBioscience) and Cy3-conjugated secondary antibodies. After counterstained with 4,6-diamidino-2-phenylindole (DAPI), cells were observed under a fluorescence microscope (Leica, Germany).

\subsubsection{Flow Cytometry Analysis}

For surface markers characterization, cells were stained with fluorescent monoclonal mouse anti-human antibodies containing CD34-PE, and CD45-PE, CD73-PE, CD29-APC, CD90-FITC, CD105-PerCP-Cy5.5 ${ }^{\mathrm{TM}}$ (BD Biosciences, USA). Fluorescence analysis was evaluated in flow cytometer (Thermo Fisher Scientific).

\subsubsection{Colony Forming Assay}

After 10 days of culture, cells were stained with $0.1 \%$ toluidine blue (Sigma Aldrich, MO, USA).

\subsubsection{Chondrogenic Differentiation}

SCAPs were cultured in chondrogenic medium for 1 month. After that, cells were immersed in paraformaldehyde for 48 hours, then imbedded in paraffin, and sliced into $5 \mu \mathrm{m}$ sections. Then, cells were stained with Alcian blue staining for 30 min.

\subsubsection{Osteogenic differentiation}

Cells were seeded in 12-well plates. Afterward, mineral induction medium consisting of $\beta$ glycerophosphate10 mM, ascorbate-2-phosphate50 mM, and dexamethasone $100 \mathrm{nM}$ (Sigma), 10\% FBS and $1 \%$ Pen-Strep was added to the wells. The culture plates were incubated in humidified condition for 21 days.

\subsubsection{Adipogenic Differentiation}

SCAPs were cultured in adipogenic medium (Cyagen, China) for 28 days. After stained with Oil Red 0 reagent (Cyagen) for $5 \mathrm{~min}$, lipid droplets were observed under microscope.

\subsection{Transfection}


The miR-24-3p oligos (inhibitor, mimic, and inhibitor NC and mimic NC) and control siRNA (NC), siRNA for MACF1(siMACF1) were designed by Ribio (Ribio CO., China). Cells were transfected with oligos and siRNAs via riboFECTTM CP following the manufacturer's protocols. Western blot and qRT-PCR were used to verify the interference efficiency. Lentiviral vectors overexpressing MACF1 were constructed and produced by Shanghai Genechem Company (Shanghai, China). SCAPs were inoculated overnight and infected with lentiviruses with polybrene.

\subsection{Cell Counting Kit-8 Assay (CCK-8)}

SCAPs were respectively transfected with miR-124-3p. 1 inhibitor, mimic, inhibitor NC and mimic NC for 48 h. Next, cells were seeded on 96-well plates and cultured for 0, 1, 3, 5 and $7 \mathrm{~d}$. Afterward, $10 \mu \mathrm{L}$ CCK-8 (Dojindo, Japan) and $90 \mu \mathrm{L}$ a-MEM were added to each well for $2 \mathrm{~h}$. The optical densities (OD) were measured at $450 \mathrm{~nm}$.

\subsection{5-Ethynyl-2冈-Deoxyuridine (EdU) assay}

The EdU DNA Proliferation Detection kit (Ribo Biotechnology, China) was used to detect cell proliferation ratio. After culture, cells were fixed and administrated with EdU labeling solution. Cells were photographed under inverted fluorescence microscope (Leica, Germany).

\subsection{Alizarin red staining}

After washed and fixed, SCAPs were stained with ARS solution (Sigma, USA). The red sediments of calcium deposition were observed under microscope. For quantitative analysis, mineralized nodules were dissolved by $10 \%$ cetylpyridinium chloride (CPC; Sigma, USA). Then OD value was measured at $562 \mathrm{~nm}$.

\subsection{Alkaline phosphatase activity}

Cells were harvested and permeated in Triton X-100. After centrifugation, the supernatant transferred into fresh $1.5 \mathrm{ml}$ tubes with relative working solution and then were incubated. The OD value was measured at $520 \mathrm{~nm}$.

\subsection{ALP staining}

After washed and fixed, cells were stained using the BCIP/NBT ALP staining kit (Beyotime, China). Cells were observed with microscope.

\subsection{RNA extraction and quantitative real- time polymerase chain reaction (qRT-PCR)}

RNA isolation was performed using the TRIzol ${ }^{\text {TM }}$ (Invitrogen) method. The cDNAs were synthesized and used for PCR. qRT-PCR reactions were performed by SYBR Green method. GAPDH and U6 were used as internal controls. Bulge-Loop miRNA qPCR Primer kit (RiboBio) was used for measuring miRNA-124.3p.1 expression. Primers in this study are listed in Table 1. 


\subsection{Western blotting}

RIPA buffer was used to extract proteins with protease inhibitor cocktail. After PAGE, the proteins were blotted onto PVDF membranes. Membranes were blocked, incubated with specific primary antibody and next day with the secondary HRP-conjugated antibody

\subsection{Immunofluorescence staining}

Cells were fixed, permeabilized. Then, cells were blocked and incubated with primary antibodies, fluorescent dye-labeled designated secondary antibody and DAPI above

\subsection{Luciferase Assay}

The potential binding site of MACF1-wt and mutant sequence MACF1-mut was synthesized into pmiRGLO (Promega, Madison, WI, USA). SCAPs were co-transfected with the MACF1-wt or MACF11-mut reporter gene plasmid and miR-124-3p.1 mimic. The activities were measured by a Promega luciferase assay (Promega, USA) were normalized against the activity of the Renilla luciferase gene.

\subsection{Co-immunoprecipitation (Co-IP) assay}

After cells lysed and centrifuged, supernatants were incubated with the anti-MACF1 antibody in rotation. Next day, the supernatants were mixed with protein G plus A agarose (Beyotime, China) at in rotation. Then, the immunocomplexes were washed. Beads complexes were resuspended in $2 \times$ loading buffer.

\subsection{Animal procedures}

SCAPs with miR-124-3p. 1 mimic and miR-124-3p. 1 mimic NC induced in MM for 7 days were harvested for the in vivo study. About $6.0 \times 10^{6}$ SCAPs were mixed with Bio-Oss Collagen scaffolds (Geistlich, Germany). And then the mixtures were implanted into the dorsal surface of BALB/c homozygous 5-weekold nude mice. Two months later, the implants were harvested and were detected under micro-CT analysis. After that, implants were decalcified and embedded with paraffin. Paraffin sections were stained with hematoxylin and eosin (H\&E) staining and Masson's trichrome staining.

\subsection{Statistical analysis}

All data are expressed as the mean \pm SD and were analyzed by one-way analysis of variance (ANOVA). $p<0.05$ was considered statistically significant.

\section{Results}

\subsection{Characterization of SCAPs}

Primary SCAPs were cultured after 3 days (Figure 1A above). SCAPs were displayed long spindle shape at passage 3 (Figure 1A below). IF staining showed SCAPs were stained positively for MSCs surface 
molecule STRO-1 (Figure 1B). SCAPs positively expressed the mesenchymal stem cell markers CD73, CD90, CD29 and CD105, but negatively expressed the hematopoietic cell markers CD45, CD34 (Figure 1C). Colony-forming assays were conducted to detect the colony forming efficiency. The result showed single colony-forming unit of SCAPs (Figure 1D). The results of staining showed that SCAPs had the potential of differentiation into chondrocytes osteoblasts and adipocytes (Figure 1E, F, G).

\section{2 miR-124-3p.1 was downregulated and MACF1 was upregulated during mineralization induction of SCAPS}

The expression of miR-124-3p.1 was markedly downregulated in SCAPs after 7days of mineralization induction, but the MACF1 expression showed an inverse trend (Figure. 2A, B). To detect the effect of miR124-3p. 1 on differentiation, SCAPs were transfected with miR-124-3p. 1 mimic, mimic NC, inhibitor, inhibitor NC. Transfection efficacy in SCAPs was verified by qRT-PCR. The miR-124-3p.1 level was dramatically increased in miR-124.3p.1 mimic, whereas decreased in miR-124.3p.1 inhibitor group, compared with relative NC groups (Figure 2C, D). CCK-8 assay and EdU staining were conducted to detect the effect of miR-124-3p.1 on the proliferation of SCAPs. The result of CCK-8 showed that knockdown of miR-124-3p.1 showed no significant influence on the proliferation of SCAPs (Figure 2E). There was also no significant difference between miR-124-3p.1 mimic NC and miR-124-3p.1 mimic groups (Figure 2F). EdU assay showed that there was no significant difference on the DNA synthesis in miR-124-3p.1 inhibitor NC group and miR-124-3p.1 inhibitor group (Figure 2G, I). Moreover, miR-124-3p.1 overexpression has no significant influence on the proliferation of SCAPs (Figure $2 \mathrm{H}, \mathrm{J}$ ).

\subsection{Effect Of Mir-124-3p.1 On Differentiation Of Scaps}

ALP staining and ALP activity assay showed increased ALP activity after the knockdown of miR-124-3p.1 but demonstrated decreased ALP activity after overexpression of miR-124-3p.1 (Figure 3A, B). Similarly, ARS staining showed that miR-124-3p.1 knockdown promoted mineralized nodules formation in SCAPs, whereas the formation of mineralized nodules was negatively mediated by upregulation of miR-124-3p.1 in SCAPs (Figure 3C, D). In addition, qRT-PCR analysis showed that the expression of DSPP, RUNX2, ALP and OSX mRNA had all showed a significant increase in miR-124-3p.1 knockdown group compared to control group, while the expression of DSPP, RUNX2, ALP and OSX mRNA in miR-124-3p.1 overexpression group was dramatically decreased (Figure 3E). Western blot analysis showed that miR-124-3p.1 knockdown enhanced the odontogenic and osteogenic differentiation related proteins (DSPP, RUNX2, ALP and OSX). Overexpression of miR-124-3p.1 in SCAPs could significantly decrease the expression of odontogenic and osteogenic differentiation markers (DSPP, RUNX2, ALP and OSX) (Figure 3F, G). Immunofluorescence staining indicated that miR-124-3p.1 knockdown significantly up-regulated the levels of DSPP, RUNX2, ALP in SCAPs (Figure 3H, J, L). Conversely, miR-124-3p.1 overexpression remarkably down-regulated the expressions of DSPP, RUNX2, ALP (Figure 31, K, M). SCAPs stably expressing miR-124-3p.1 mimic and miR-124-3p.1 mimic NC loaded on Bio-Oss Collagen scaffolds were 
implanted in immunocompromised mice subcutaneously (Figure 4A). MicroCT imaging showed BV/TV in miR-124-3p.1 mimic group was less than miR-124-3p.1 mimic group (Figure 4B). Masson and H\&E staining showed less bone-like structures formed in in the presence of mimics for miR-124-3p.1 in SCAPs than the control group (Figure. 4C, D). These results indicate that miR-124-3p.1 negatively regulated osteogenic and odontogenic differentiation of SCAPs.

\subsection{Macf1 Was The Target Of Mir-124-3p.1}

Subsequently, the potential binding sequences between miR-124-3p.1 and MACF1 were predicted by miRanda (Figure 5A). Dual-Luciferase reporter gene assay showed that co-transfection of miR-124-3p.1 mimic and MACF1 3'-UTR WT could remarkably decrease the Luciferase activity (Figure 5B). Hence, the binding relationship of MACF1 to miR-124-3p.1 was verified. The overexpression of miR-124a-3p.1 in SCAPs significantly upregulated the mRNA and protein levels of MACF1. However, miR-124a-3p.1 knockdown could up-regulate its expression level (Figure 5C, D, E). Immunofluorescence staining showed the same trend above (Figure 5F). These results provided strong evidence that MACF1 was the target of miR-124-3p.1.

\subsection{Silencing of MACF1 inhibited the odontogenic and osteogenic differentiation of SCAPs, overexpression of MACF1 promoted the odontogenic and osteogenic differentiation of SCAPs}

To explore the potential influence of MACF1 on the osteogenic and odontogenic differentiation of SCAPs, MACF1 knockdown and MACF1 overexpression were established. qRT-PCR showed that a significant decrease of MACF1 expression in siMACF1 group and an obvious increase in the MACF1 overexpression group compared to relative control groups (Figure 6A). Therefore, western blot analysis confirmed the protein expression level of MACF1 was significantly downregulated in siMACF1 group and was dramatically upregulated in MACF-1-over group (Figure 6B, C). ALP staining and ALP activity assay showed that ALP activity in siMACF1 group was clearly decreased and ALP activity were obviously enhanced in the MACF1 overexpression group (Figure 6D, E). ARS staining and relevant CPC quantitative analysis showed matrix mineralization was dramatically downregulated in siMACF1 group and a contrary trend was detected in MACF-1 overexpression group (Figure 6F, G). Besides, qRT-PCR and western blot showed that deletion of MACF1 downregulated the expression of DSPP, RUNX2, ALP and OSX. MACF1 overexpression increased gene and protein expression associated with osteo/odontogenetic differentiation (DSPP, RUNX2, ALP and OSX) (Figure 6H, I, J). These results indicated that MACF1 downregulation is negatively associated with the osteogenic and odontogenic differentiation in SCAPs. But MACF1 overexpression promoted the osteogenic and odontogenic differentiation of SCAPs in vitro. 


\subsection{Downregulation of miR-124-3p.1 enhanced odontogenic and osteogenic differentiation via inhibiting MACF1 expression}

To investigate the mechanism of miR-124-3p.1 in regulating odontogenic and osteogenic differentiation, SCAPs were transfected with miR-124-3p. 1 inhibitor NC, miR-124-3p.1 inhibitor or miR-124-3p.1 inhibitor+ NC, miR-124-3p.1 inhibitor+ siMACF1, respectively. The result of qRT-PCR demonstrated that miR-1243p.1 knockdown could upregulate the expression of MACF1, while siMACF1 rescued the above changes induced by miR-124-3p.1 inhibitor, causing the decreased level of MACF1 (Figure 7A). In consonance with the results of qRT-PCR, western blot and immunofluorescence staining showed that upregulation of MACF1 induced by miR-124-3p.1 inhibitor could be negatively regulated by knockdown of MACF1 (Figure 7B, C, D). Analogously, the relative expression levels of DSPP, RUNX2, ALP and OSX were significantly upregulated after miR-124-3p.1 inhibitor. However, the levels of these above genes were markedly inhibited by MACF1 knockdown (Figure 7E). In addition, western blot showed that downregulation of miR-124-3p.1 remarkably promoted the expression of DSPP, RUNX2, ALP and OSX. Meanwhile, the expression of DSPP, RUNX2, ALP and OSX was reversed by co-transfection of miR-124-3p.1 inhibitor and siMACF1 (Figure 7F, $\mathrm{G})$. Immunofluorescence staining further verified the above results (Figure $7 \mathrm{H}, \mathrm{I}, \mathrm{J}$ ). Our data suggested that MACF1 repression was able to partially inhibited the positive effect of miR-124-3p.1 downregulation on osteogenic and odontogenic differentiation.

\subsection{Overexpression of miR-124-3p.1 inhibited odontogenic and osteogenic differentiation via mediating MACF1 expression}

To further explore the mechanism of miR-124-3p.1 in regulating odontogenic and osteogenic differentiation, miR-124-3p.1 mimic and MACF1-over lentivirus were transfected, respectively. The result of qRT-PCR showed that MACF1 was markedly decreased by overexpression of miR-124-3p.1, which could be reversed by MACF1 overexpression (Figure 8A). Western blot and immunofluorescence staining showed that downregulation of MACF1 induced by miR-124-3p.1 mimic could be significantly reversed by overexpressing MACF1 (Figure 8B, C, D). Moreover, MACF1 overexpression also reversed the variation of the relative expression levels of DSPP, RUNX2, ALP and OSX inhibited by the miR-124-3p.1 mimic (Figure 8E). Western blot showed that upregulation of miR-124-3p.1 also decreased the expression of DSPP, RUNX2, ALP and OSX. Meanwhile, the expression of DSPP, RUNX2, ALP and OSX was reversed by cotransfection of miR-124-3p.1 mimic and MACF1-over lentivirus (Figure 8F, G). Immunofluorescence staining further verified the above results (Figure $8 \mathrm{H}, \mathrm{I}, \mathrm{J}$ ). Our data suggested that MACF1 overexpression was able to partially enhance the inhibitory effect of miR-124-3p.1 upregulation on osteogenic and odontogenic differentiation. 


\subsection{Macf1 Interacts With Smad7 In Scaps}

To further explore the mechanism that MACF1 regulates SCAPs' differentiation, western blot and CO-IP were conducted. The result of western blot showed that MACF1 knockdown not only significantly decreased expression of smad7 in the cytoplasm, but also inhibited the expression of smad7 in the nucleus (Figure 9A, C). Next, we found that smad7 were increased in SCAPs as compared with NC-over cells, the enhancement was especially obvious in the nucleus (Figure 9B, D). We found that smad7 were co-immunoprecipitated with MACF1 using the antibody recognizing MACF1 (anti-MACF1). Co-IP result showed that smad7 was detectable in the anti-MACF1 immunoprecipitated products, indicating that MACF1 can interact with smad7 in SCAPs. Moreover, MACF1 is related to smad7 nuclear translocation (Figure 9E). The mechanism diagram in this study was showed in Figure 9F.

\section{Discussion}

Multiple studies have demonstrated that dental apical papillae directly contribute toward the formation of the tooth roots[35]. Furthermore, SCAPs are an effective cell resource for tissue regeneration[36]. In the present study, we found that miR-124-3p.1 inhibited the osteo-/odontogenic differentiation potential of SCAPs. Importantly, we found that miR-124-3p.1 played an inhibitory role in the differentiation of SCAPs by binding to the 3 '-UTR of MACF1, further regulation of smad7 entry into the nucleus.

Recent evidence indicates that miRNAs play important role in osteoblast differentiation and bone formation[37]. Previous studies have demonstrated that miRNAs act as important regulators for the stemness of oral mesenchymal stem cells[37,38]. Recently, the critical role of miR-124-3p.1 in osteogenic differentiation of human BMSCs has been previously demonstrated[34]. We, therefore, assume that miR124-3p. 1 can inhibit osteogenic and odontogenic differentiation of SCAPs through the MACF1/smad7 axis. Our study showed that overexpression of miR-124-3p.1 decreased the odonto/osteogenic differentiation of SCAPs while miR-124-3p.1 inhibition elevated osteogenic and odontogenic differentiation. The function and molecular mechanisms of miR-124-3p.1 in osteogenic and odontogenic differentiation was remains unclear. According to bioinformatics prediction (TargetScan V7.2), we discovered that MACF1 was a predicted target gene of miR-124-3p.1. As a member of the spectraplakin family of cytoskeletal crosslinking proteins, MACF1 is widely expressed in different tissues[39]. Accumulating evidence indicates that MACF1 is a critical factor in modulating actin and microtubule cytoskeletal networks and regulating cytoskeletal distribution, cell migration, cell survival and cell differentiation[40]. It was reported that MACF1 correlates with various physiological and pathological processes[41]. MACF1 has a significant effect on osteogenesis the differentiation of primary osteoblasts[42]. MACF1 is an important regulator in various signal transduction and cellular processes[43]. There are reports showed that downregulation of MACF1 suppressed the differentiation of osteoblastic cell line[44, 45]. Taken together, the evidence illustrated that MACF1 can regulate the differentiation of osteoblast via Wnt signaling[46]. Moreover, smad7 is identified as a new downstream 
target of MACF1 and MACF1 promotes bone formation by facilitating smad7 nuclear translocation[47]. Previously, smad7 acts as an inhibitor to inhibit bone formation by a negative feedback way[48]. However, recent studies have emphasized the positive role of smad7 in stem cells such as osteoblasts and myoblasts $[49,50]$. It is also reported that nucleus smad7 can enhance osteogenic differentiation potential[ $[47,49]$.

\section{Conclusion}

In summary, we found that miR-124-3p.1 negatively regulated the osteogenic and odontogenic differentiation potential by negatively regulating MACF1/smad7 axis in SCAPs. Additional studies are required to further confirm whether miR-124-3p.1 and MACF1 may enhance odontogenesis of SCAPs in vivo. Further studies are also needed to confirm the relationship between smad7 and differentiation of SCAPs, which will provide a theoretical basis for treating dental diseases.

\section{Declarations}

\section{Acknowledgements}

Not applicable. We would like to give our sincere appreciation to the reviewers for their helpful comments on this article.

\section{Conflict of interest}

Authors declare no conflicts of interest.

\section{Authors Contributions}

$\mathrm{LN}$ conceived and designed the experiments, collected and assembled data, and wrote the manuscript. LZH, YM and GYC performed data analysis and interpretation. WYQ, WJT and YCT collected and analyzed data. XT and FL reviewed the manuscript. YJH conceived and designed the study, provided financial support and study material, performed the data analysis and interpretation, and approved the final version of the manuscript. All authors read and approved the manuscript.

\section{Funding}

This work was supported by the National Natural Science Foundation of China (grant numbers: 81900962 and 82170940$)$.

\section{Availability of data and materials}

Datasets used and analyzed during the current study are available from the corresponding author on reasonable request. 
Studies were carried out in accordance with the Declaration of Helsinki and got the approval of the Ethical Committee of Nanjing Medical University.

\section{Consent for publication}

Not applicable.

\section{References}

1. Xueying Zhuang LJ, Huan Jiang, Yao Liu, Xuemei Liu, Jing Bi, Weidong Zhao, Zhenjiang Ding, and Xu Chen. Exosomes Derived from Stem Cells from the Apical Papilla Promote Dentine-Pulp Complex Regeneration by Inducing Specific Dentinogenesis. Stem Cells International. 2020; 2020: 5816723.

2. Shi Yu JL, Yuming Zhao, Xiaoxia Li, Lihong Ge. Comparative Secretome Analysis of Mesenchymal Stem Cells From Dental Apical Papilla and Bone Marrow During Early Odonto/Osteogenic Differentiation: Potential Role of Transforming Growth Factor- $\beta 2$. Frontiers in Physiology. 2020; 11: 41.

3. Haoqing Yang GL, Nannan Han, Xiuli Zhang, Yangyang Cao, Yu Cao, Zhipeng Fan. Secreted frizzledrelated protein 2 promotes the osteo/odontogenic differentiation and paracrine potentials of stem cells from apical papilla under inflammation and hypoxia conditions. Cell Proliferation. 2020; 53(1): e12694.

4. Haoqing Yang YC, Jianpeng Zhang, Yuncun Liang, Xiaomin Su, Chen Zhang, Huina Liu, Xiao Han, Lihua Ge, Zhipeng Fan. DLX5 and HOXC8 enhance the chondrogenic differentiation potential of stem cells from apical papilla via LINC01013. Stem Cell Reseach Therapy. 2020;11(1): 271.

5. Ana Paula Aquistapase Dagnino PCC, Renata Priscila Medeiros, Marina Estrázulas, Luiza Wilges Kist, Maurício Reis Bogo, João Batista Blessmann Weber, Maria Martha Campos, and Jefferson Braga Silva. Neural Regenerative Potential of Stem Cells Derived from the Tooth Apical Papilla. Stem Cells Development. 2020; 29(23): 1479-96.

6. Nathaniel S Hwang CZ, Yong-Sung Hwang, Shyni Varghese. Mesenchymal stem cell differentiation and roles in regenerative medicine. Wiley Interdisciplinary Reviews. System Biology and Medicine. 2009; 1(1): 97-106.

7. Christian Morsczeck GS, Torsten Eugen Reichert, Florian Völlner, Kerstin Galler, Oliver Driemel. Somatic stem cells for regenerative dentistry. Clinical Oral Investigations. 2008; 12(2): 113-8.

8. Gianrico Spagnuolo BC, Massimo Marrelli, Carlo Rengo, Sandro Rengo, Marco Tatullo. Commitment of Oral-Derived Stem Cells in Dental and Maxillofacial Applications. Dentistry Journal. 2018; 6(4): 72.

9. Cyclic Adenosine Monophosphate Promotes Odonto/Osteogenic Differentiation of Stem Cells from the Apical Papilla via Suppression of Transforming Growth Factor Beta 1 Signaling. Journal of Endodontics. 2019; 45(2): 150-5.

10. José Luis Sanz LF, Alicia Almudéver, Julia Guerrero-Gironés and Carmen Llen. Viability and Stimulation of Human Stem Cells from the Apical Papilla (hSCAPs) Induced by Silicate-Based 
Materials for Their Potential Use in Regenerative Endodontics: A Systematic Review. Materials (Basel). 2020 Feb; 13(4): 974. 2020; 13(4): 974.

11. Jun Kang WF, Qianyi Deng, Hongwen He, Fang Huang. Stem Cells from the Apical Papilla: A Promising Source for Stem Cell-Based Therapy. Biomedicine Research International. 2019; 2019: 6104738.

12. Wei F YS, Guo Q, Zhang X, Ren D, Lv T, Xu X. MicroRNA-21 regulates Osteogenic Differentiation of Periodontal Ligament Stem Cells by targeting Smad5. Scientific Reports. 2017; 7(1): 16608.

13. P De Berdt JV, B Ucakar, L Elens, A Diogenes, J G Leprince 1, R Deumens, A des Rieux. Dental Apical Papilla as Therapy for Spinal Cord Injury. Journal of Dental Research. 2015; 94(1575-1581).

14. Ana L Morgado CMPR, Susana Solá. MicroRNA-145 Regulates Neural Stem Cell Differentiation Through the Sox2-Lin28/let-7 Signaling Pathway. Stem Cells. 2016; 34(5): 1386-95.

15. Zhangyuan Lin HH, Min Wang, Jieyu Liang. MicroRNA-130a controls bone marrow mesenchymal stem cell differentiation towards the osteoblastic and adipogenic fate. Cell Proliferation. 2019; 52(6): e12688.

16. Christina Schreck RI, Christoph Ziegenhain, Theresa Sippenauer, Franziska Ruf, Lynette Henkel, Florian Gärtner, Beate Vie, M Carolina Florian, Nicole Mende, Anna Taubenberger, Áine Prendergast, Alina Wagner, Charlotta Pagel, Sandra Grziwok, Katharina S Götze, Jochen Guck, Douglas C Dean, Steffen Massberg, Marieke Essers, Claudia Waskow, Hartmut Geiger, Mathias Schiemann, Christian Peschel, Wolfgang Enard, Robert A J Oostendorp. Niche WNT5A regulates the actin cytoskeleton during regeneration of hematopoietic stem cells. The Journal of Experimental Medicine. 2017; 14(1): 165-81.

17. Y-P Lin L-ML, Q-H Liu, Y Ni, Y Zhong, S Yu. MiRNA-128-3p induces osteogenic differentiation of bone marrow mesenchymal stem cells via activating the Wnt3a signaling. European Rev iew for Medican and Pharmacological Science. 2021; 25(3): 1225-32.

18. Kyoung Min Kim S-KL. Role of miRNAs in bone and their potential as therapeutic targets. Current Opinion in Pharmacology. 2014; 16: 133-41.

19. Chi-Chih Chang MTV, Li Chen, Nicholas Ditzel, Dang Q.S. Le, Philipp Dillschneider, Moustapha Kassem and Jørgen Kjems. Global MicroRNA Profiling in Human Bone Marrow Skeletal-Stromal or Mesenchymal-Stem Cells Identified Candidates for Bone Regeneration. Molecular Therapy. 2018; 26(2): 593-605.

20. Fang Li DL, Meng Zhang, Junwei Sun, Wenting Li, Ruirui Jiang, Ruili Han, Yanbin Wang, Yadong Tian, Xiangtao Kang, Guirong Sun. miRNA-223 targets the GPAM gene and regulates the differentiation of intramuscular adipocytes. Gene. 2019; 685(106-113).

21. Christoph Klingelhöffer CC, Tobias Ettl, Torsten Reichert, Christian Morsczeck. miRNA-101 supports the osteogenic differentiation in human dental follicle cells. Archives of Oral Biology. 2016; 72: 47-50.

22. Zhihao Chen YZ, Chao Liang, Lei Chen, Ge Zhang, Airong Qian. Mechanosensitive miRNAs and bone formation. Internatinal Journal of Molecular Science. 2017; 18(8): 1684. 
23. Rijiang Chen HQ, Yan Tong, Fake Liao, Xiunian Hu, Yongrong Qiu, Yuanjun Liao. MiRNA-19a-3p alleviates the progression of osteoporosis by targeting HDAC4 to promote the osteogenic differentiation of hMSCs. Biochemical and Biophysical Reseach Communication. 2019; 516(3): 66672.

24. S Vimalraj NS. MicroRNAs: synthesis, gene regulation and osteoblast differentiation. Current Issues in Molecular Biology. 2013; 15: 7-18.

25. D-W Ge W-WW, H-T Chen, L Yang, X-J Cao. Functions of microRNAs in osteoporosis. European Review for Medical and Pharmacological Science. 2017; 21(21): 4784-9.

26. Yin Tang LZ, Tianchi Tu, Yijia Li, Dana Murray, Qisheng Tu, Jake Jinkun Chen. MicroRNA-99a is a novel regulator of KDM6B-mediated osteogenic differentiation of BMSCs. Journal of Cellular and Molecular Medicine. 2018; 22(4): 2162-76.

27. Tilde Eskildsen HT, Jan Stenvang, Basem M Abdallah, Nicholas Ditzel, Anne Yael Nossent, Mads Bak, Sakari Kauppinen, Moustapha Kassem. MicroRNA-138 regulates osteogenic differentiation of human stromal (mesenchymal) stem cells in vivo. Proceedings of the National Academy of Sciences of the United States of America. 2011; 108(15): 6139-44.

28. Abdul S Qadir SU, Heesu Lee, Kyunghwa Baek, Byoung Moo Seo, Gene Lee, Gwan-Shik Kim, Kyung Mi Woo, Hyun-Mo Ryoo, Jeong-Hwa Baek. miR-124 negatively regulates osteogenic differentiation and in vivo bone formation of mesenchymal stem cells. Journal of Cell Biochemistry. 2015; 16(5): 730-42.

29. Yanqiu Wang XP, Jintao Wu, Lin Jin, Yan Yu, Romila Gobin, Jinhua Yu. MicroRNA hsa-let-7b suppresses the odonto/osteogenic differentiation capacity of stem cells from apical papilla by targeting MMP1. Journal of Cellular Biochemistry. 2018; 119(8): 6545-54.

30. Guiling Yan YL, Lu Zhan, Shuhan Sun, Jihang Yuan, Tiantian Wang, Yupeng Yin, Zhihui Dai, Yiqing Zhu, Zhijing Jiang, Lin Liu, Yinxing Fan, Fu Yang, Wei Hu. Decreased miR-124-3p promoted breast cancer proliferation and metastasis by targeting MGAT5. American Journal of Cancer Reseach. 2019; 9(3): 585-96.

31. Di Hu ML, Jing Su, Keke Miao, Xinguang Qiu. Dual-targeting of miR-124-3p and ABCC4 promotes sensitivity to adriamycin in breast cancer cells miR-124, a potential therapeutic target in colorectal cancer. Genetic Testing and Molecular Biomarkers. 2019; 23(3): 156-65.

32. P Wang L-DZ, M-C Sun, W-D Gu, H-Z Geng. Over-expression of mir-124 inhibits MMP-9 expression and decreases invasion of renal cell carcinoma cells. European Review for Medical Pharmacology Science. 2018; 22(19): 6308-14.

33. Xiaohong Deng YC, Zhao Liu, Jingning Xu. MiR-124-3p.1 Sensitizes Ovarian Cancer Cells to Mitochondrial Apoptosis Induced by Carboplatin. OncoTargets and Therapy. 2020; 13: 5375-86.

34. Yujing Cao QL, Yang Li. Astragaloside IV Improves Tibial Defect in Rats and Promotes Proliferation and Osteogenic Differentiation of hBMSCs through MiR-124-3p.1/STAT3 Axis. Journal of Nature Products. 2021; 84(2): 287-97. 
35. Wen Zhang XZ, Junyuan Li, Jianmao Zheng, Xiaoli Hu, Meng Xu, Xueli Mao, Junqi Ling. Foxc2 and BMP2 Induce Osteogenic/Odontogenic Differentiation and Mineralization of Human Stem Cells from Apical Papilla. stem cells International. 2018; 25(2018): 2363917.

36. Sijia Na HZ, Fang Huang, Weiqi Wang, Yin Ding, Dechao Li, Yan Jin. Regeneration of dental pulp/dentine complex with a three-dimensional and scaffold-free stem-cell sheet-derived pellet. Journal of Tissue Engineering Regeneration Medicine. 2016; 10(3): 261-70.

37. Lei Zhu Z-WL, Gang Wang, Hong Zhang, Ben Liu, Qing-Jia Xu. MicroRNA-495 downregulates AQP1 and facilitates proliferation and differentiation of osteoblasts in mice with tibial fracture through activation of p38 MAPK signaling pathway. Science Reports. 2019; 9(1): 16171.

38. Yao S ZW, Ou Q, Liang L, Lin X, Wang Y. MicroRNA-214 Suppresses Osteogenic Differentiation of Human Periodontal Ligament Stem Cells by Targeting ATF4. Stem Cells Int. 2017; 2017: 3028647.

39. Jeffrey J. Moffat MK, Eui-Man Jung, Amanda L. Smith and Woo-Yang Kima. The role of MACF1 in nervous system development and maintenance. Seminars in Cell Developmental Biology. 2017; 69: 9-17.

40. Lifang Hu YX, Zhipeng Xiong, Fan Zhao, Chong Yin, Yan Zhang, Peihong Su, Dijie Li, Zhihao Chen, Xiaoli Ma, Ge Zhang, Airong Qian. MACF1, versatility in tissue-specific function and in human disease. Semininars in Cell Developmental Biology. 2017; 69: 3-8.

41. Peihong Su CY, Dijie Li, Chaofei Yang, Xue Wang, Jiawei Pei, Ye Tian, Airong Qian. MACF1 promotes preosteoblast migration by mediating focal adhesion turnover through EB1. Biology Open. 2020; 9(3): bio048173.

42. Xiao Lin YX, Zhihao Chen, Jianhua Ma, Wuxia Qiu, Kewen Zhang, Fang Xu, Kai Dang, Airong Qian. Microtubule actin crosslinking factor 1 (MACF1) knockdown inhibits RANKL-induced osteoclastogenesis via Akt/GSK3ß/NFATc1 signalling pathway. Molecular and Cellular Endocrinology. 2019; 494: 110494.

43. Chong Yin YT, Lifang Hu, Yang Yu, Zixiang Wu, Yan Zhang, Xue Wang, Zhiping Miao and Airong Qqian. MACF1 alleviates aging-related osteoporosis via HES1. Journal of Cellular and Molecular Medicine. 2021; 25(13): 6242-57.

44. Lifang Hu PS, Chong Yin, Yan Zhang, Runzhi Li, Kun Yan, Zhihao Chen, Dijie Li, Ge Zhang, Liping Wang, Zhiping Miao, Airong Qian, Cory J Xian. Microtubule actin crosslinking factor 1 promotes osteoblast differentiation by promoting beta-catenin/TCF1/Runx2 signaling axis. Journal of Cell Physiology. 2018; 233(2): 1574-84.

45. Yan Zhang CY, Lifang Hu, Zhihao Chen, Fan Zhao, Dijie Li, Jianhua Ma, Xiaoli Ma, Peihong Su, Wuxia Qiu, Chaofei Yang, Pai Wang, Siyu Li, Ge Zhang, Liping Wang, Airong Qian, Cory J Xian. MACF1 Overexpression by Transfecting the $21 \mathrm{kbp}$ Large Plasmid PEGFP-C1A-ACF7 Promotes Osteoblast Differentiation and Bone Formation. Human Gene Therapy. 2018; 29(2): 259-70.

46. Lifang Hu PS, Runzhi Li, Chong Yin, Yan Zhang, Peng Shang, Tuanmin Yang, Airong Qian. Isoforms, structures, and functions of versatile spectraplakin MACF1. BMB Reports. 2016; 49(1): 37-44. 
47. Fan Zhao XM, Wuxia Qiu, Pai Wang, Ru Zhang, Zhihao Chen, Peihong Su, Yan Zhang, Dijie Li, Jianhua Ma, Chaofei Yang, Lei Chen, Chong Yin, Ye Tian, Lifang Hu, Yu Li, Ge Zhang, Xiaoyang Wu, Airong Qian. Mesenchymal MACF1 Facilitates SMAD7 Nuclear Translocation to Drive Bone Formation. Cells. 2020; 9(3): 616.

48. Keiji Miyazawa KM. Regulation of TGF- $\beta$ Family Signaling by Inhibitory Smads. Cold Spring Harbors Perspectives in Biology. 2017; 9(3): a022095.

49. Nan Li WY-WL, Si-En Lin, Ming Ni, Ting Zhang, Xiao-Ru Huang, Hui-Yao Lan, Gang Li. Partial loss of Smad7 function impairs bone remodeling, osteogenesis and enhances osteoclastogenesis in mice. Bone. 2014; 67: 46-55.

50. Tetsuaki Miyake NSA, John C McDermott. Nuclear function of Smad7 promotes myogenesis. Molecular and Cellular Biology. 2010; 30(3): 722-35.

\section{Tables}

Table 1. Primer sequences for qRT-PCR analysis of gene expression.

\begin{tabular}{|c|c|c|}
\hline Target gene & Sequences $(5 \dot{c}-3 \dot{c})$ & Product size (bp) \\
\hline \multirow[t]{2}{*}{ RUNX2 } & Forward, TCTTAGAACAAATTCTGCССTTT & 136 \\
\hline & Reverse, TGCTTTGGTCTTGAAATCACA & \\
\hline \multirow[t]{2}{*}{ OSX } & Forward, ССТССТСАGСТСАССТТСТС & 148 \\
\hline & Reverse, GTTGGGAGCCCAAATAGAAA & \\
\hline \multirow[t]{2}{*}{$A L P$} & Forward, GACCTCCTCGGAAGACACTC & 137 \\
\hline & Reverse, TGAAGGGCTTCTTGTCTGTG & \\
\hline \multirow[t]{2}{*}{ DSPP } & Forward, ATATTGAGGGCTGGAATGGGGA & 136 \\
\hline & Reverse, TTTGTGGCTCCAGCATTGTCA & \\
\hline \multirow[t]{2}{*}{ GAPDH } & Forward, GAAGGTGAAGGTCGGAGTC & \\
\hline & Reverse, GAGATGGTGATGGGATTTC & 225 \\
\hline
\end{tabular}

\section{Figures}


A

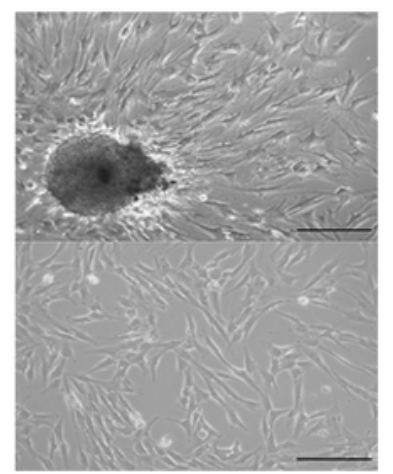

B

STRO-1/Phalloidin/DAPI

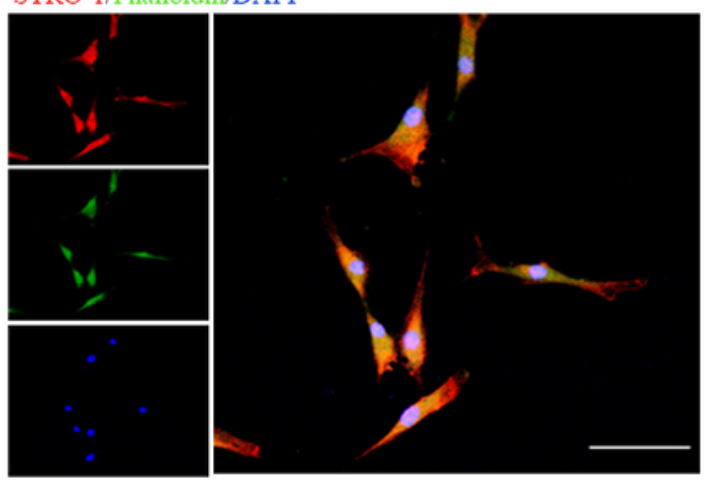

$\mathrm{C}$
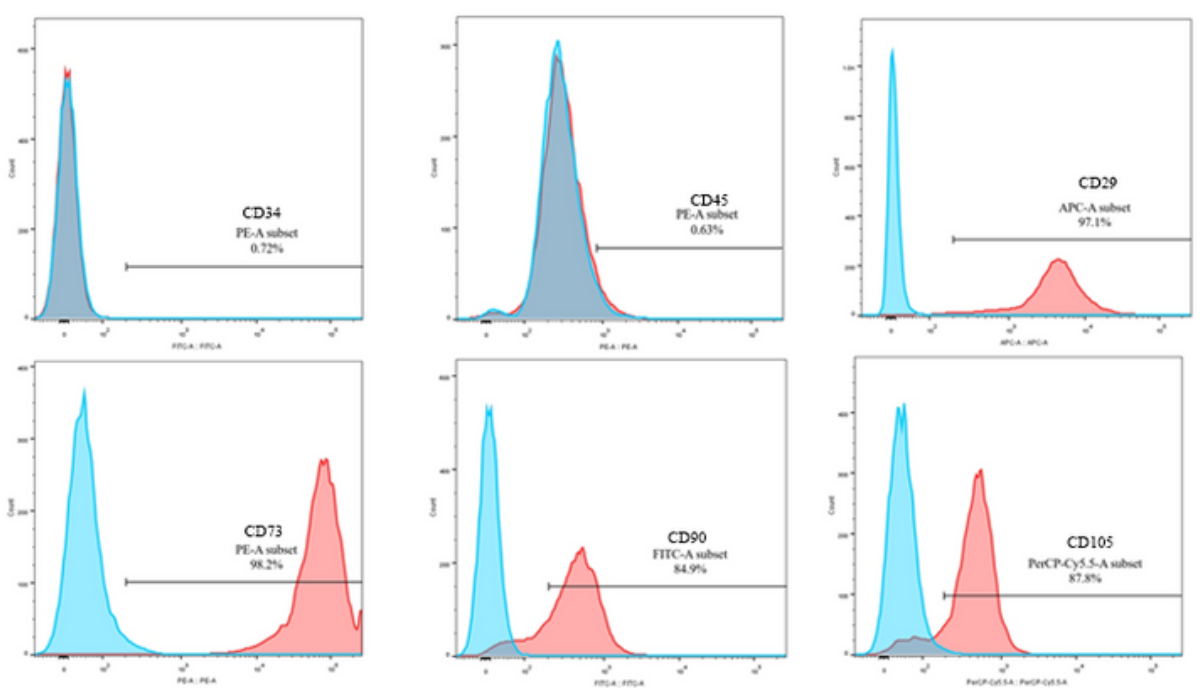

$\mathrm{D}$

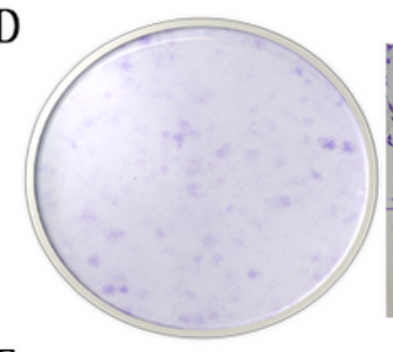

$\mathrm{F}$

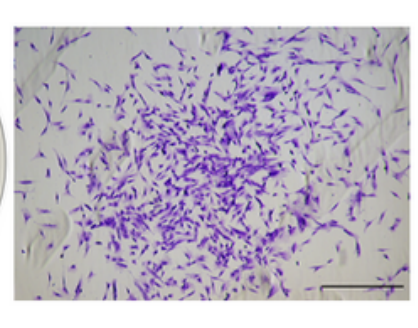

E

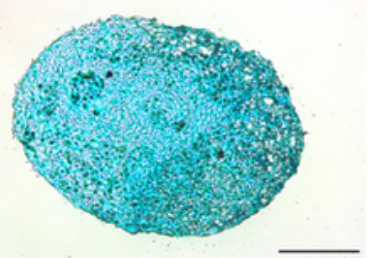

G
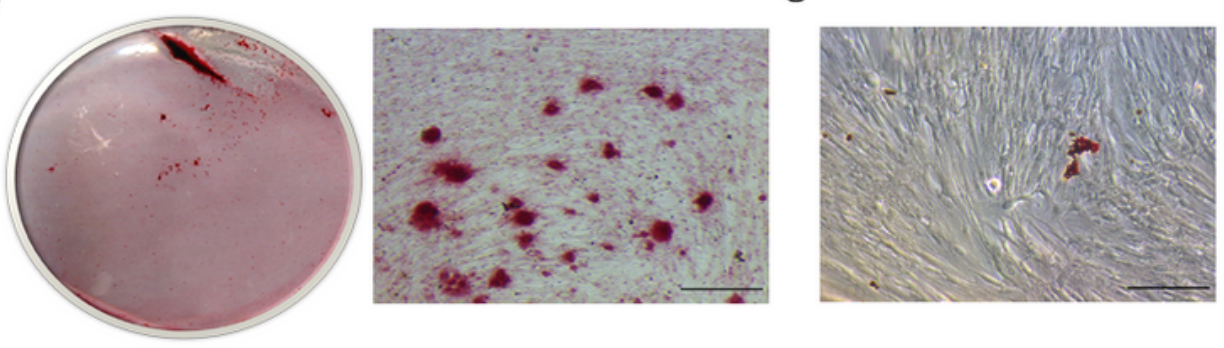

\section{Figure 1}

Characterization of SCAPs A. Primary cultured SCAPs (Above, scale bar $=200 \mu \mathrm{m}$ ); SCAPs at passage 3 (Below, scale bar $=200 \mu \mathrm{m}$ ). B. Immunofluorescence staining showed that SCAPs expressed STRO-1 (scale bar $=200 \mu \mathrm{m}$ ). C. SCAPs expressed high levels of the mesenchymal stem cell marker CD73, CD90, CD29 and CD105, but expressed low levels of the hematopoietic cell marker CD34, CD45. D. Colony forming units of hPDLSCs after cultivation (Scale bar= $200 \mu \mathrm{m}$ ). E. SCAPs had the potential of 
differentiation into chondrocytes (Scale bar $=200 \mu \mathrm{m}$ ). F. SCAPs had the potential of differentiation into osteoblasts (Scale bar= $200 \mu \mathrm{m}$ ). G. SCAPs had the potential of differentiation into adipocytes (Scale bar $=200 \mu \mathrm{m})$.

A

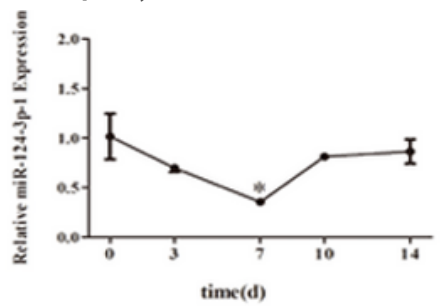

$\mathrm{C}$

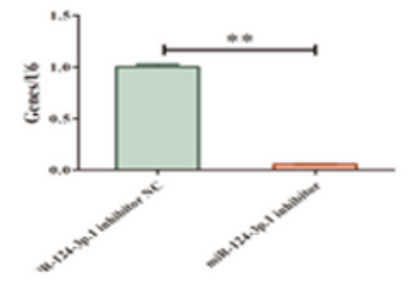

E

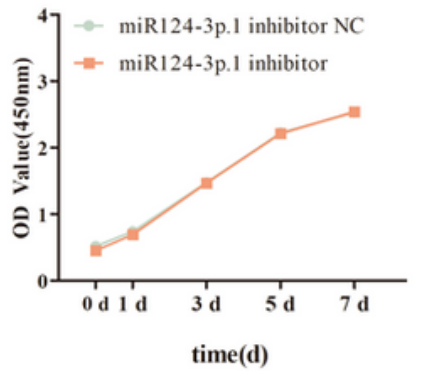

G
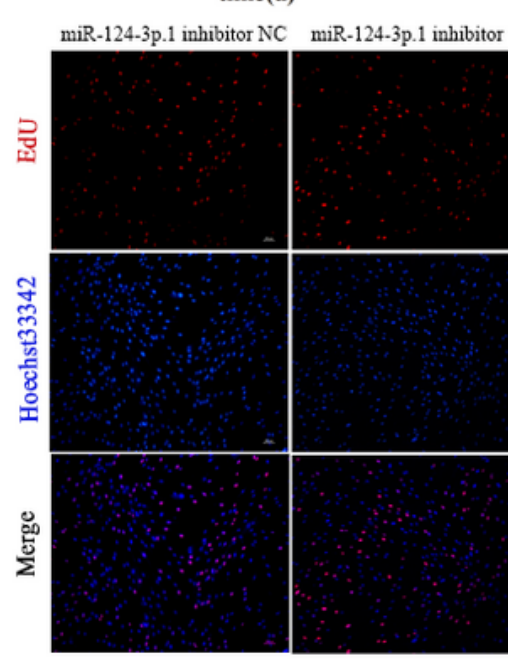

I
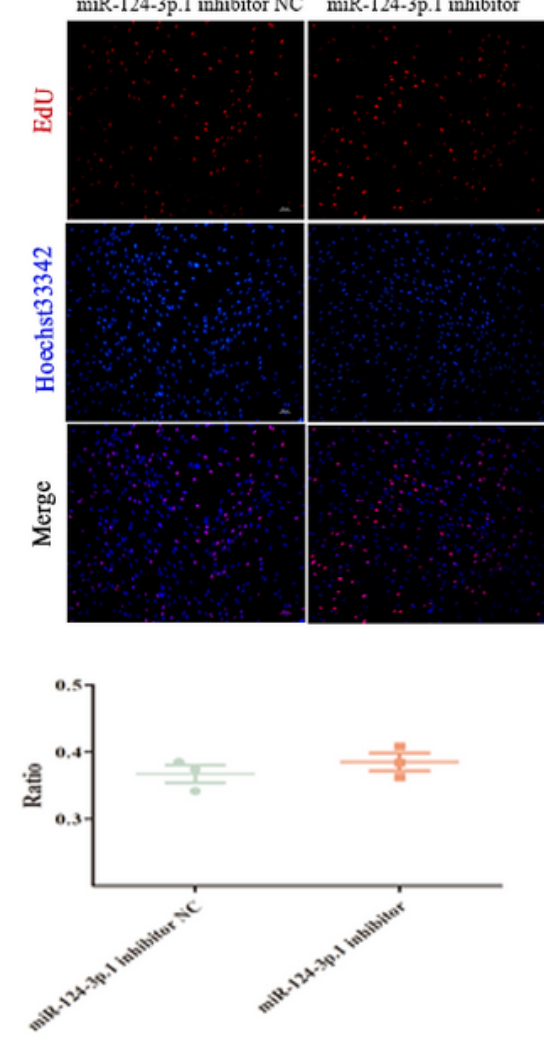

B

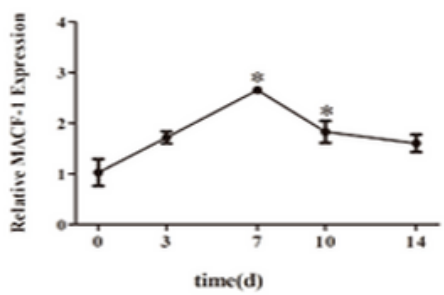

$\mathrm{D}$

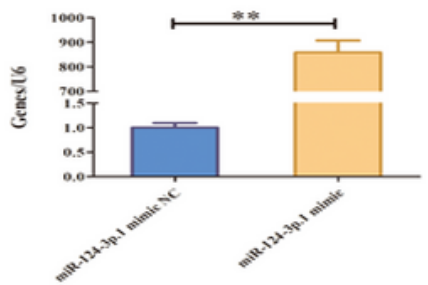

$\left.\mathrm{F} \quad{ }^{3.0}\right] \rightarrow \operatorname{miR} 124-3 \mathrm{p} .1$ mimic NC

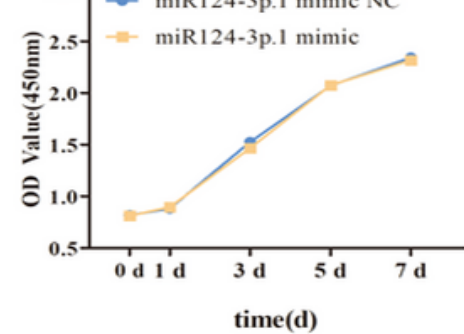

miR-124-3p.1 mimic NC

miR-124-3p.1 mimic

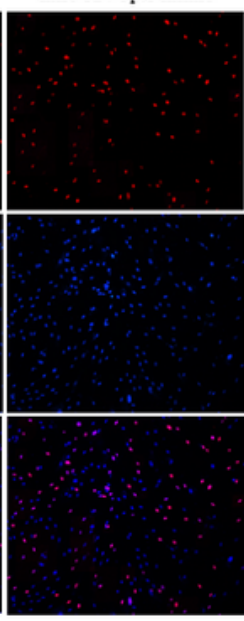

$\mathrm{J}$

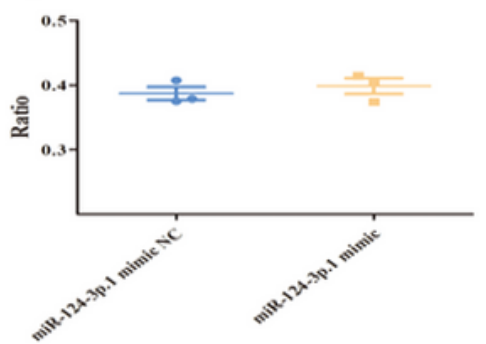

Figure 2

Effect of miR-124-3p.1 on the proliferation in SCAPs A. miR-124-3p.1 expression profile throughout differentiation induction ( ${ }^{*}<0.05, * * p<0.01$ ). B. MACF1 expression profile throughout differentiation 
induction ( $\left.{ }^{*} p<0.05,{ }^{*} p<0.01\right)$. C. The transfection effects of miR-124-3p. 1 inhibitors were confirmed by qRT-PCR $(* p<0.05, * * p<0.01)$. D. The transfection effects of miR-124-3p.1 mimics were confirmed by qRT-PCR $(* p<0.05, * \star p<0.01)$. E. Cell proliferation detected using CCK-8 assay in miR-124-3p.1 inhibitor NC, miR-124-3p.1 inhibitor groups ( $p>0.05$ ). F. Cell proliferation detected using CCK-8 assay in miR-124$3 p .1$ mimic NC, miR-124-3p.1 mimic groups (p>0.05). G. Proliferation ability assay of miR-124-3p.1 inhibitor NC, miR-124-3p.1 inhibitor groups, EdU (red) marked proliferating cells and Hoechst (blue) labeled total cell nuclei, and the red and blue images were merged to purple ones. $\mathrm{H}$. Proliferation ability assay of miR-124-3p.1 mimic NC, miR-124-3p.1 mimic groups. (I) The relative quantification of EdUpositive cells from $G(p>0.05)$. $(J)$ The relative quantification of EdU-positive cells from $H(p>0.05)$. 


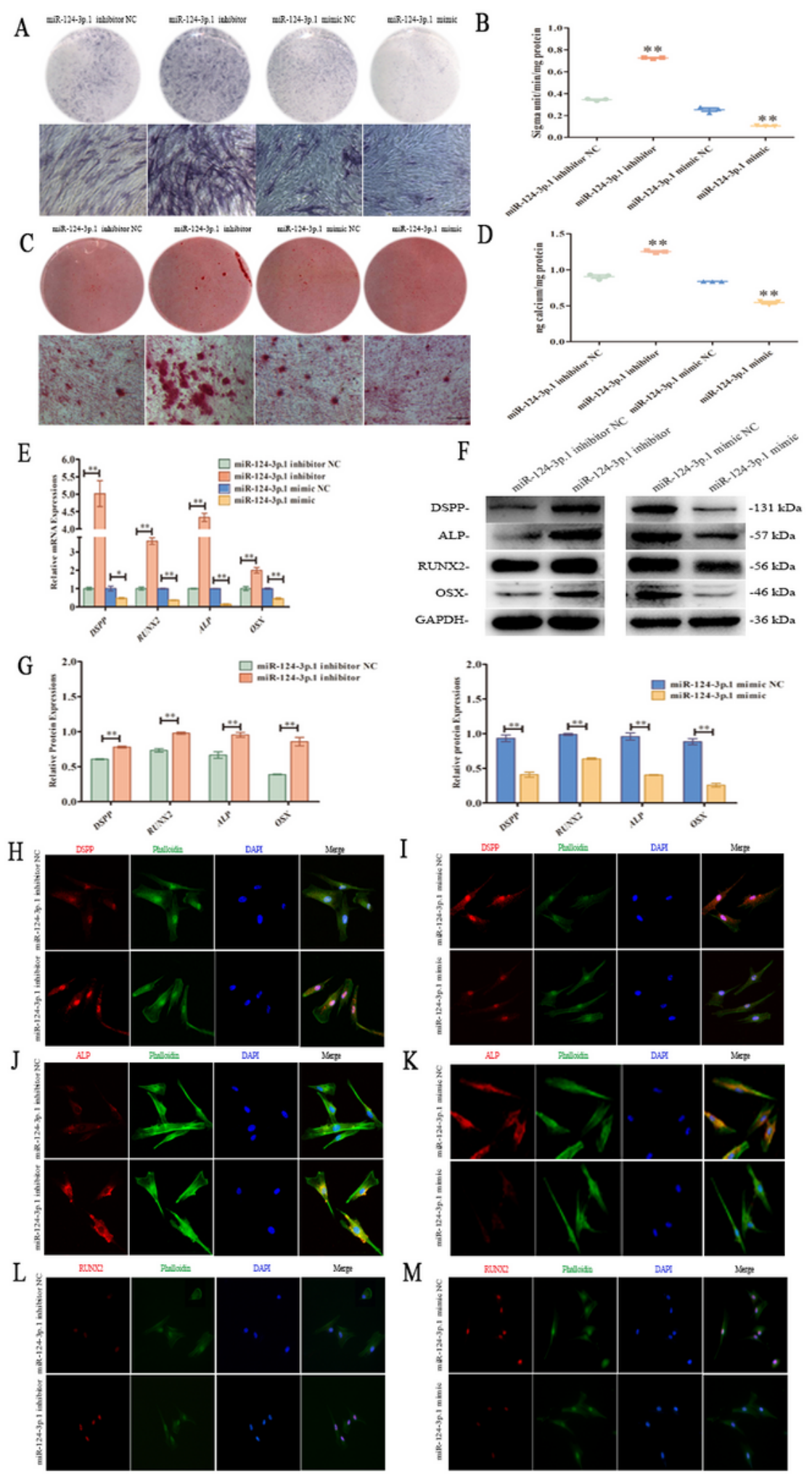

Figure 3

Effects of miR-124-3p.1 on the osteogenic and odontogenic differentiation in SCAPs A. Images of ALP staining in the miR-124-3p.1 inhibitor NC, miR-124-3p.1 inhibitor groups, miR-124-3p.1 mimic NC, miR124-3p.1 mimic groups (Scale bar= $200 \mu \mathrm{m}$ ). B. ALP activity assay was conducted in miR-124-3p.1 inhibitor NC, miR-124-3p. 1 inhibitor groups, miR-124-3p. 1 mimic NC, miR-124-3p.1 mimic groups (* $\left.p<0.05,{ }^{*} p<0.01\right)$. C. ARS staining on day 14 as indicated treatment (Scale bar $\left.=200 \mu \mathrm{m}\right)$. D. CPC 
analysis of miR-124-3p.1 inhibitor NC, miR-124-3p.1 inhibitor groups, miR-124-3p.1 mimic NC, miR-124$3 p .1$ mimic groups ( ${ }^{*}<<0.05$, $* \star p<0.01$ ). E. The expressions of DSPP, RUNX2, OSX and ALP were determined by qRT-PCR analysis ( ${ }^{*} p<0.05,{ }^{*} \mathrm{p}<0.01$ ). F. The expressions of DSPP, RUNX2, OSX and ALP were determined by western blot assay. G. Relative quantitative analysis of western blot analyses in $D(*$ $p<0.05$, ** $p<0.01) . H$, I. Immunofluorescence staining of DSPP (Scale bar= $50 \mu \mathrm{m})$. J, K.

Immunofluorescence staining of ALP (Scale bar= $50 \mu \mathrm{m}$ ). L, M. Immunofluorescence staining of RUNX2 (Scale bar $=50 \mu \mathrm{m})$.

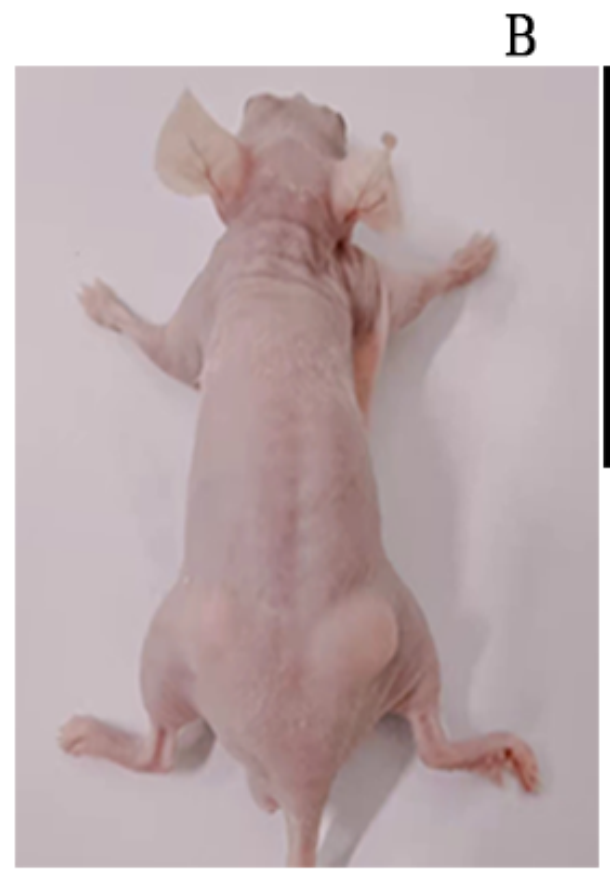

$\mathrm{C}$
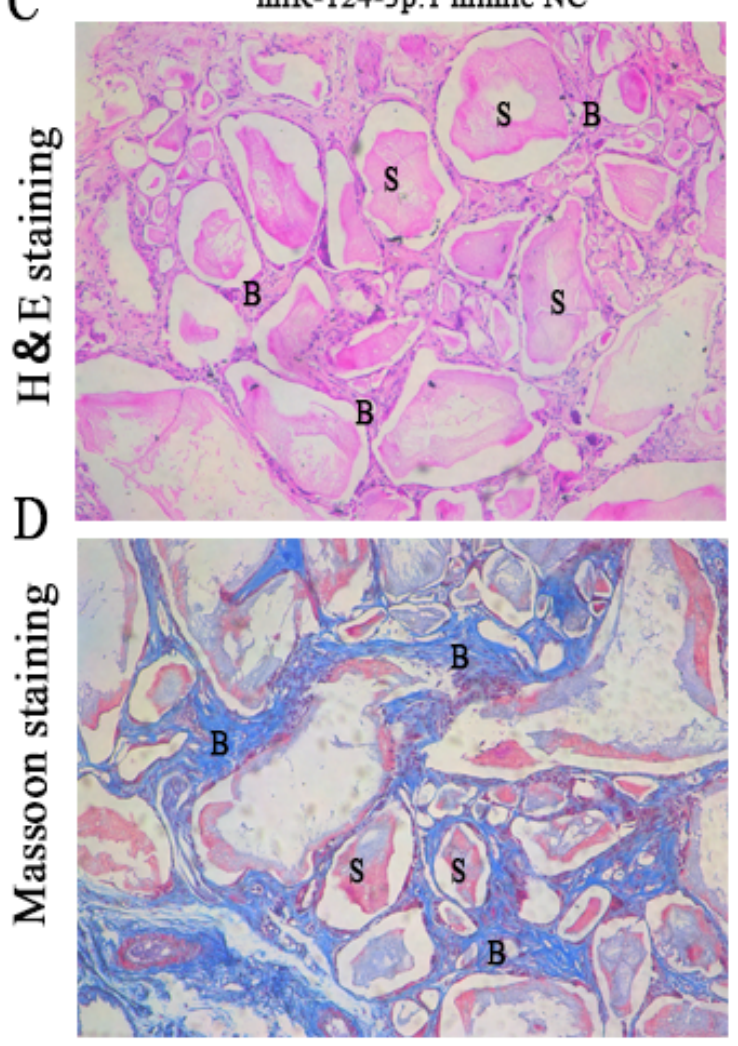

miR-124-3p.1 mimic NC
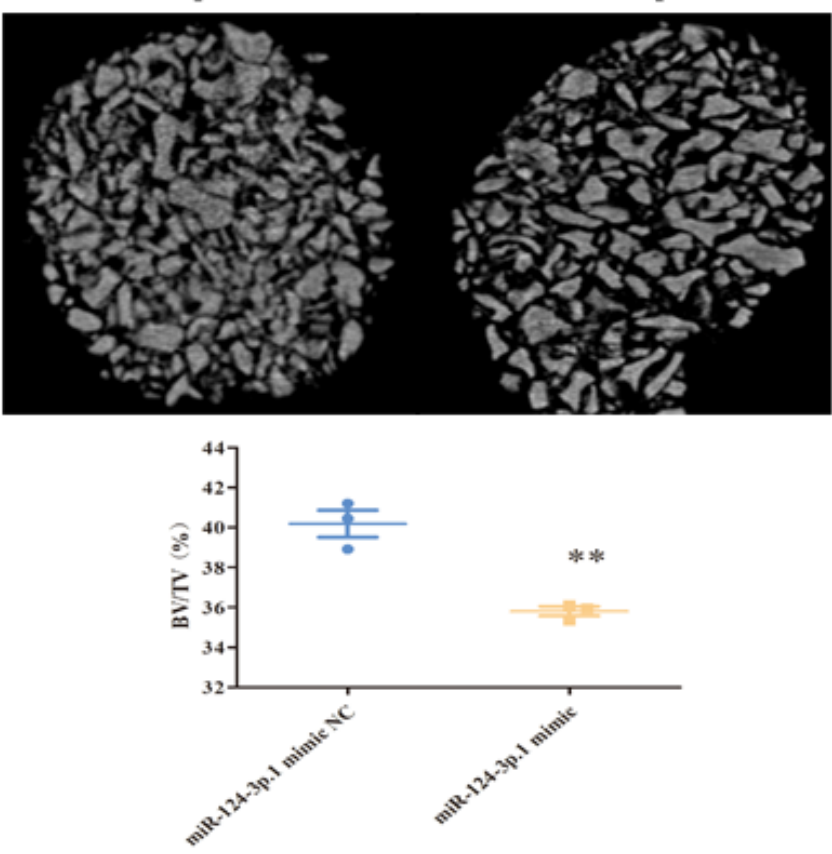

miR-124-3p.1 mimic
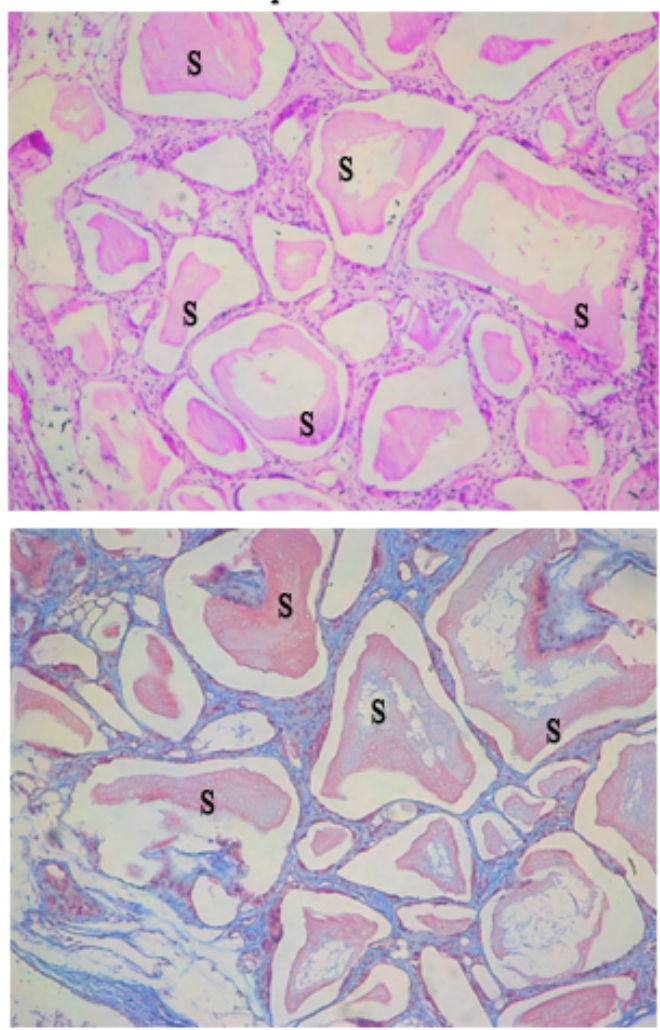


\section{Figure 4}

miR-124-3p.1 enhanced the osteo/dentinogenesis of SCAPs in vivo A. SCAPs with miR-124-3p.1 overexpression and control group were transplanted subcutaneously into nude mice. B. The results of micro-CT analyses in miR-124-3p.1 mimic group and miR-124-3p.1 mimic NC groups. C. H\&E staining of osteocalcin in miR-124-3p.1 mimic group and miR-124-3p.1 mimic NC groups. D. Masson staining of osteocalcin in miR-124-3p.1 mimic group and miR-124-3p.1 mimic NC groups.

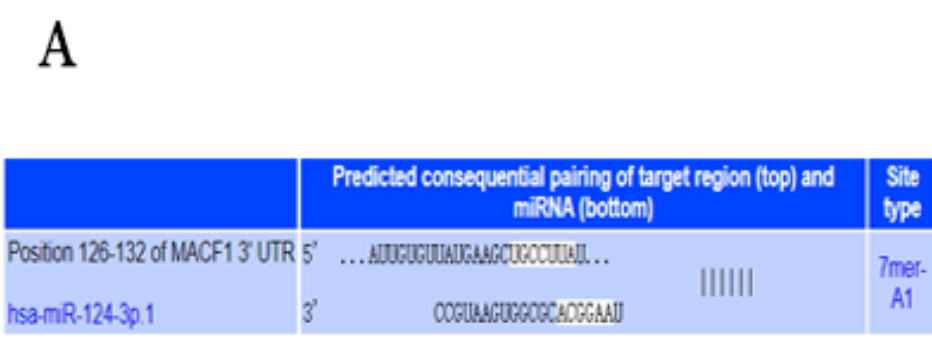

B

$\mathrm{C}$

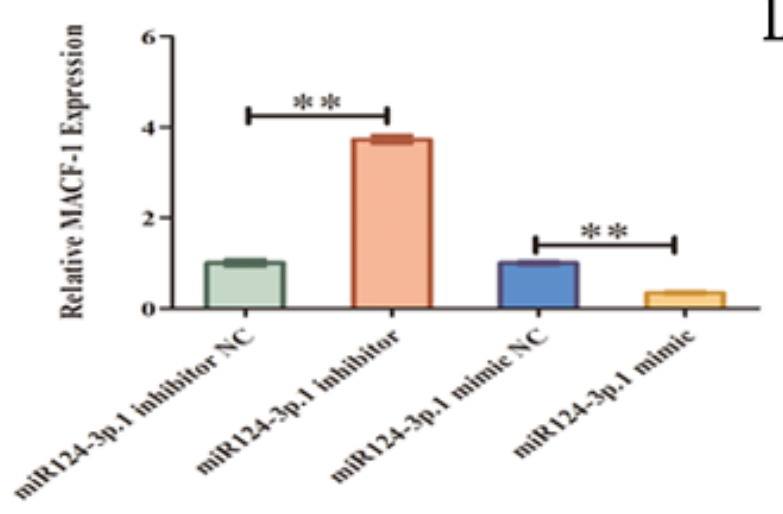

$\mathrm{D}$

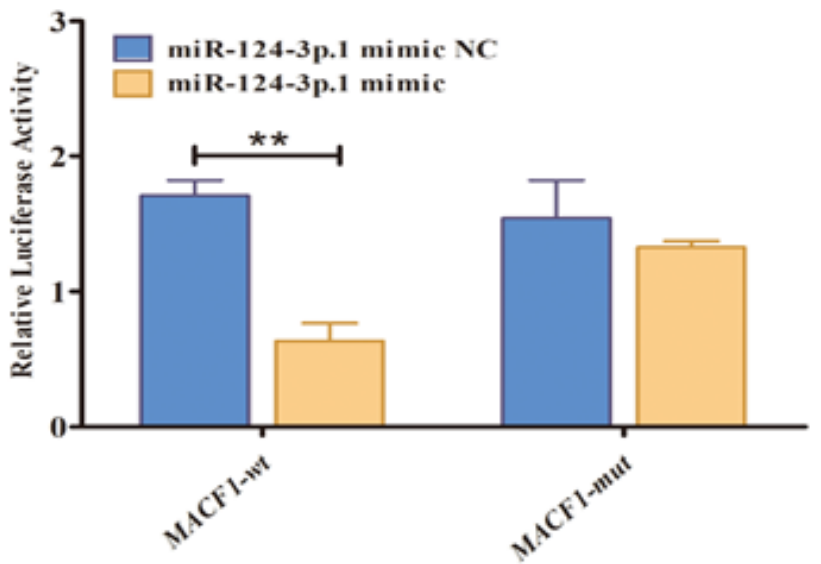

$\mathrm{E}$

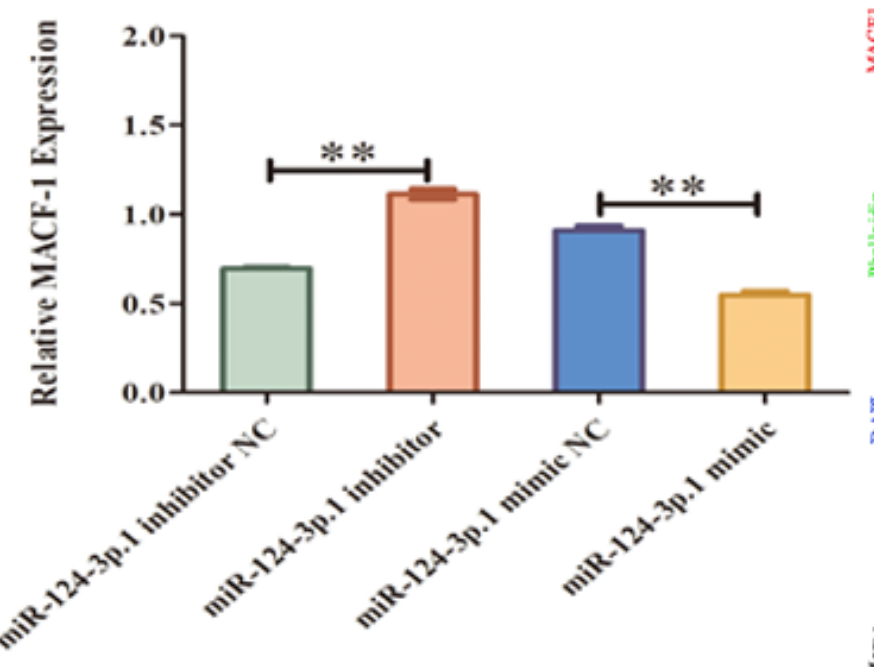

F

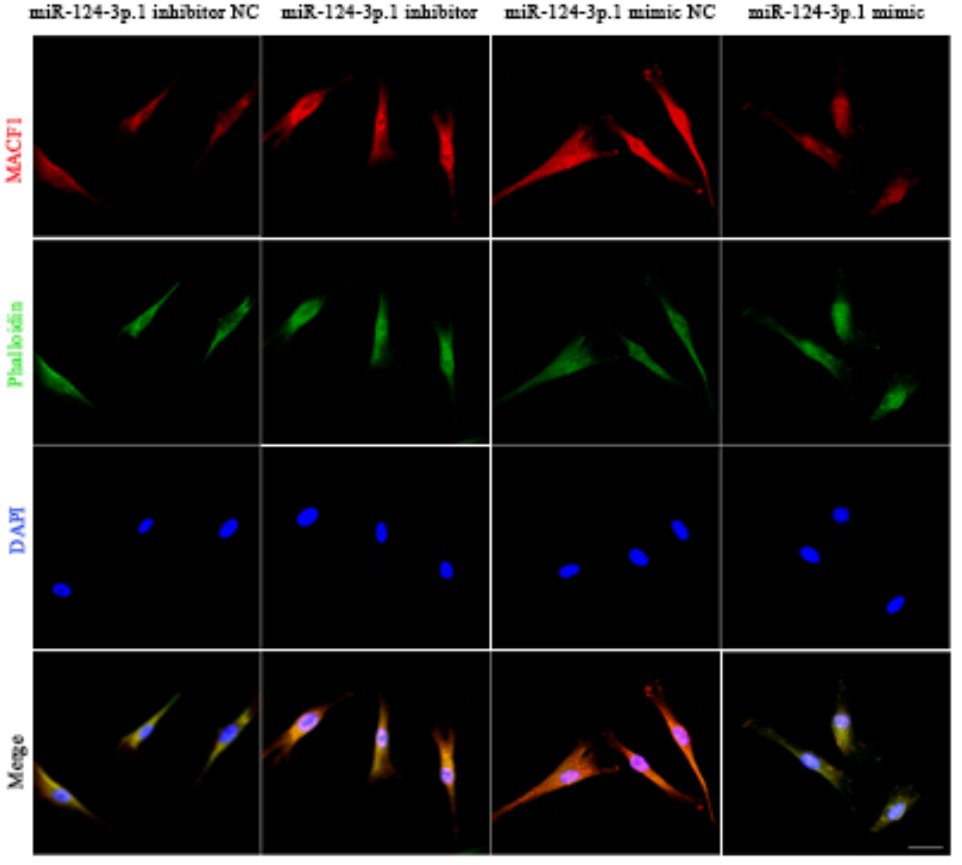




\section{Figure 5}

MACF1 is a direct target of miR-124-3p.1 A. Sequences of miR-124-3p.1 and predicted binding sites in the 3'- UTR of MACF1. B. 293T cells were transfected with either pGV272 luciferase vector containing a fragment of the MACF1 3'- UTR harboring a binding site for miR-124-3p.1, or the corresponding Mut constructs. Ectopic expression of miR-124-3p.1 led to a significant decrease of the reporter luciferase activity with the WT 3 '- UTR but not that of the Mut reporter ( $\left.{ }^{*}<0.05,{ }^{\star} * p<0.01\right)$. C. SCAPs were transfected with miR-124-3p.1 inhibitor NC, miR-124-3p.1 inhibitor, miR-124-3p.1 mimic NC and miR-124$3 p .1$ mimic. After transfection for 48 hours, the mRNA level of MACF1 was measured by qRT-PCR (* $\left.p<0.05,{ }^{*} p<0.01\right)$. D. Western blot analysis of protein expression of MACF1 and the internal control GAPDH in miR-124-3p.1 inhibitor NC, miR-124-3p.1 inhibitor, miR-124-3p.1 mimic NC and miR-124-3p.1 mimic groups. E. The quantification analysis of band intensities $\left({ }^{*} p<0.05,{ }^{\star *} p<0.01\right)$. F. Immunofluorescence staining of MACF1 (Scale bar= $50 \mu \mathrm{m}$ ). 
A

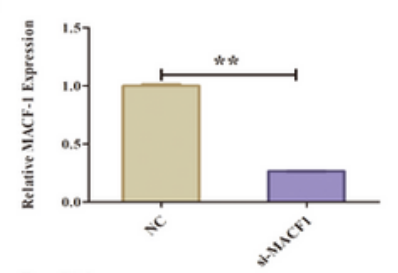

B
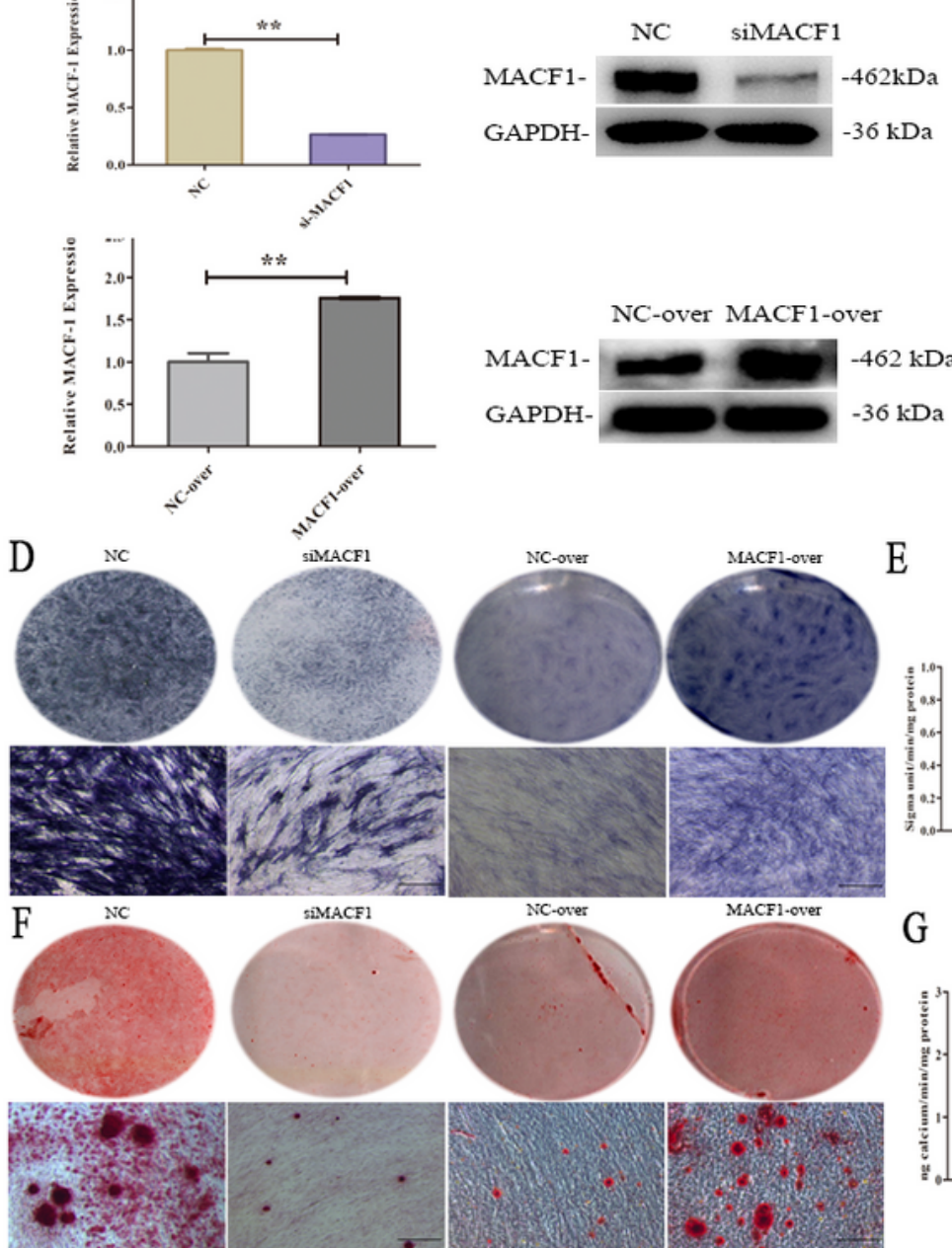

$\mathrm{H}$
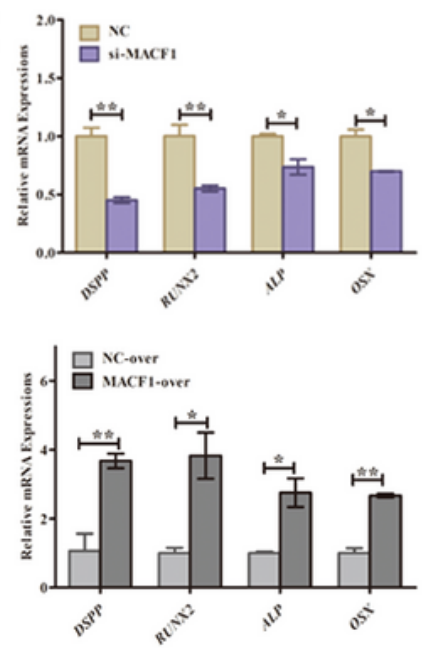
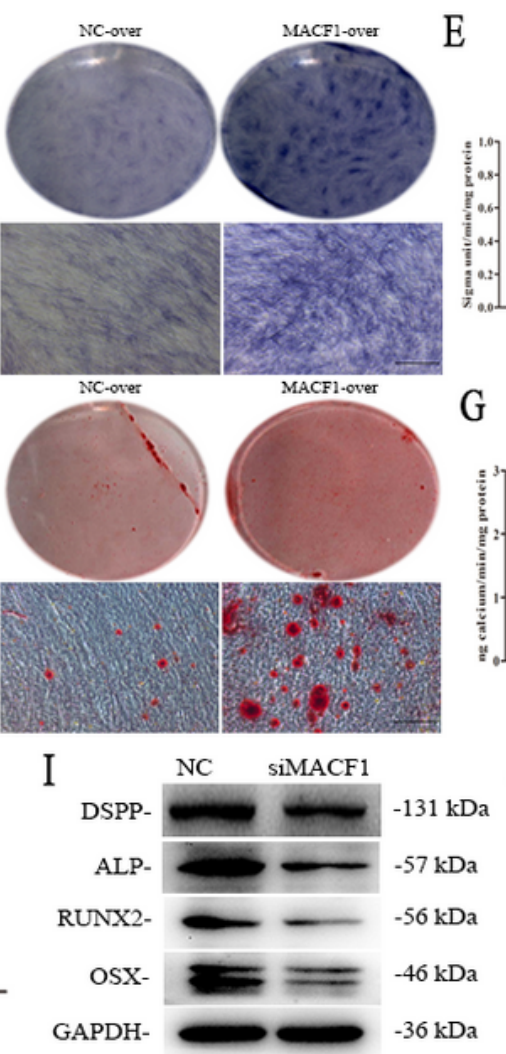

NC-over MACF1-over

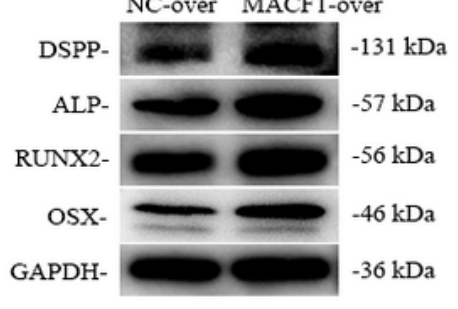

$\mathrm{C}$
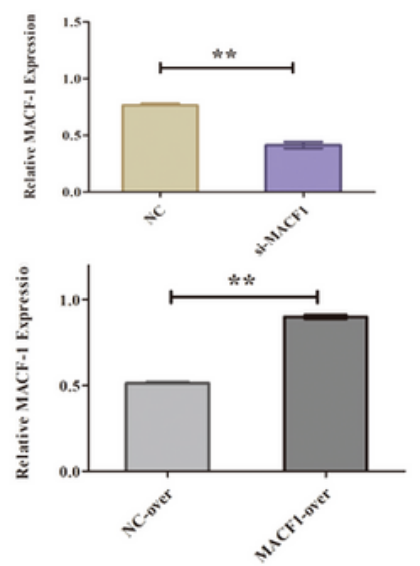

E

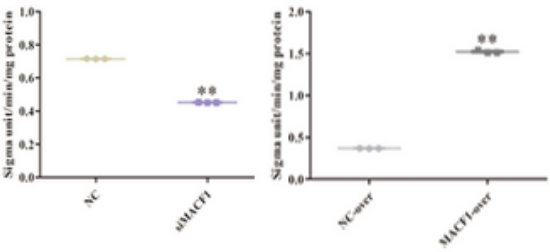

G
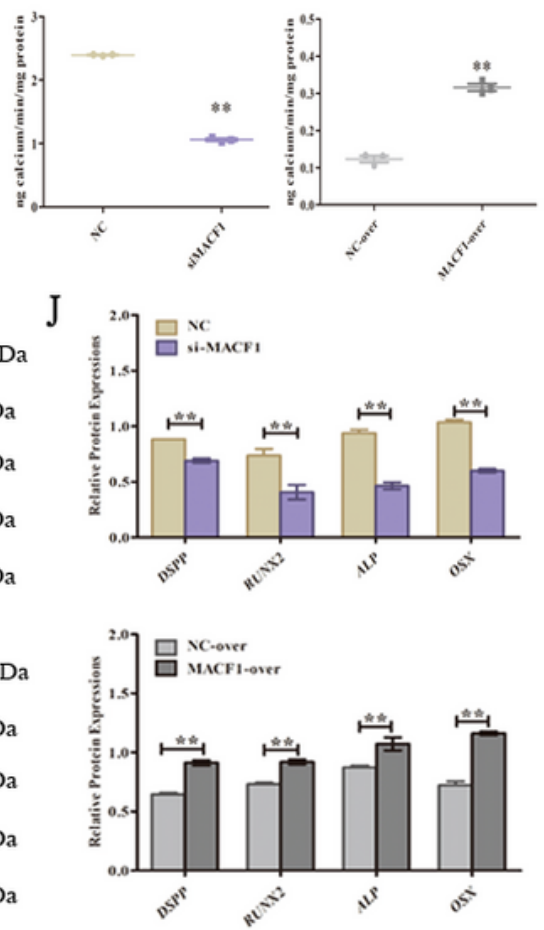

\section{Figure 6}

Knockdown of MACF1 inhibited the osteogenic and odontogenic differentiation of SCAPs,

Overexpression of MACF1 promoted the odontogenic and osteogenic differentiation of SCAPs A. qRT-PCR analysis of the expression levels of MACF1 in NC, siMACF1, NC-over and MACF1-over groups $\left({ }^{*} p<0.05\right.$, ** $p<0.01)$. B. Western blot analysis of the protein expression levels of MACF1 in NC, siMACF1, NC-over and MACF1-over groups. C. Quantification analysis of protein band in $B\left({ }^{*}<<0.05,{ }^{* *} p<0.01\right)$. D. Images of 
ALP staining in NC, siMACF1, NC-over and MACF1-over groups (Scale bar= $200 \mu \mathrm{m}$ ). E. ALP activity analysis in NC, siMACF1, NC-over and MACF1-over groups (* $p<0.05, * \star p<0.01$ ). F. The mineral nodes of SCAPs were visualized by Alizarin red staining (Scale bar $=200 \mu \mathrm{m})$. G. Calcium quantitative analysis of NC, siMACF1, NC-over and MACF1-over groups. H. qRT-PCR analysis of DSPP, RUNX2, ALP and OSX mRNA expression in SCAPs ( ${ }^{*} p<0.05,{ }^{*} p<0.01$ ). I. Western blot analysis of DSPP, RUNX2, ALP and OSX protein expression in SCAPs. J. The quantification analysis of band intensities $\left({ }^{*} p<0.05\right.$, $\left.{ }^{*} p<0.01\right)$.

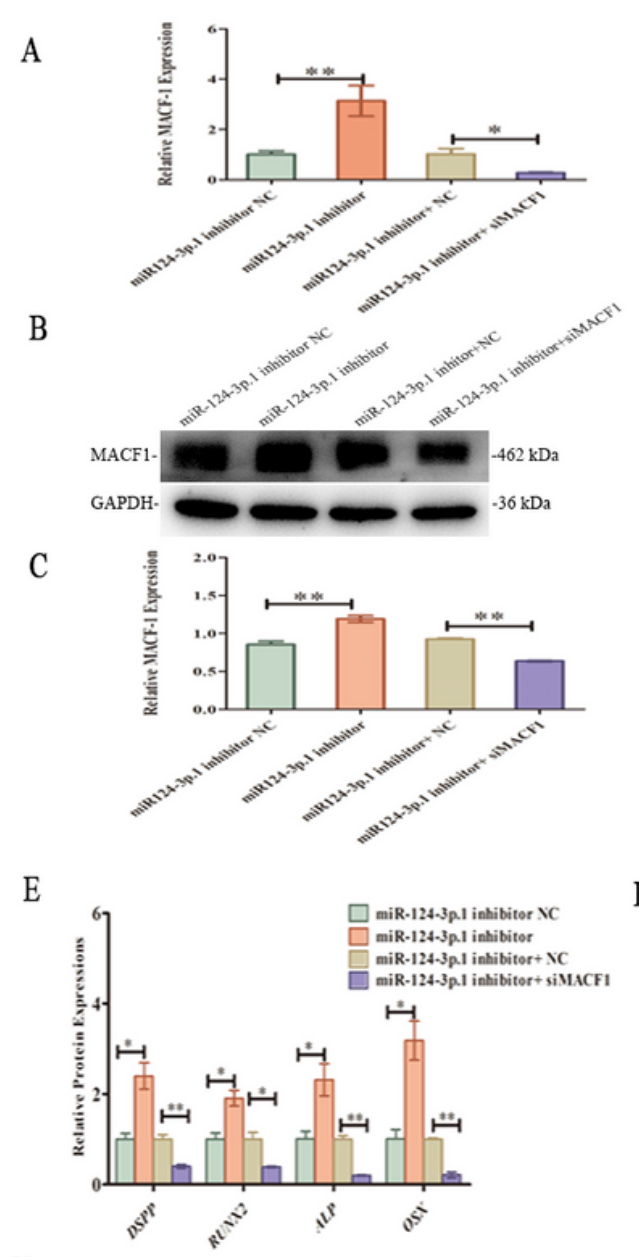

$\mathrm{H}$

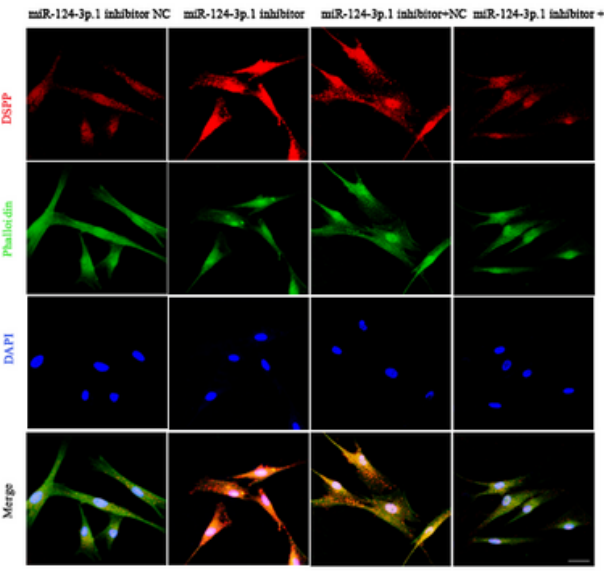

$\mathrm{D}$

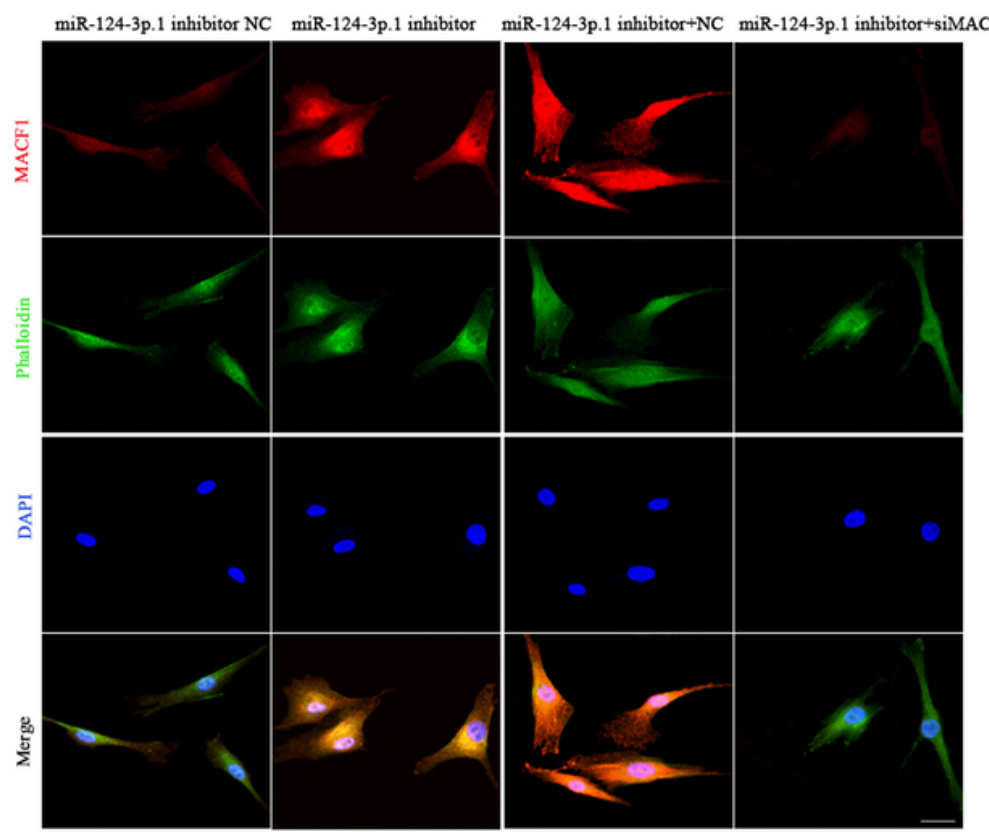

$\mathrm{F}$

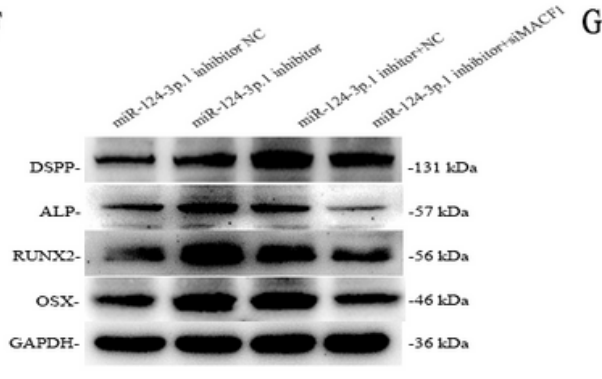

I

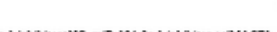

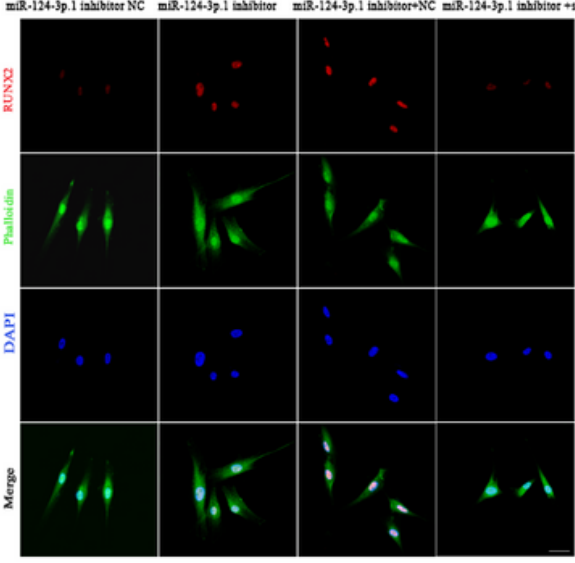

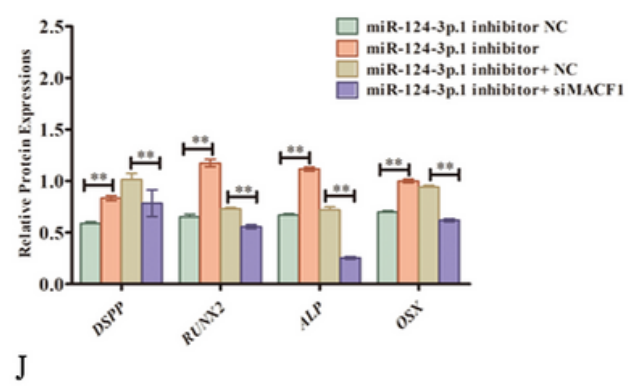

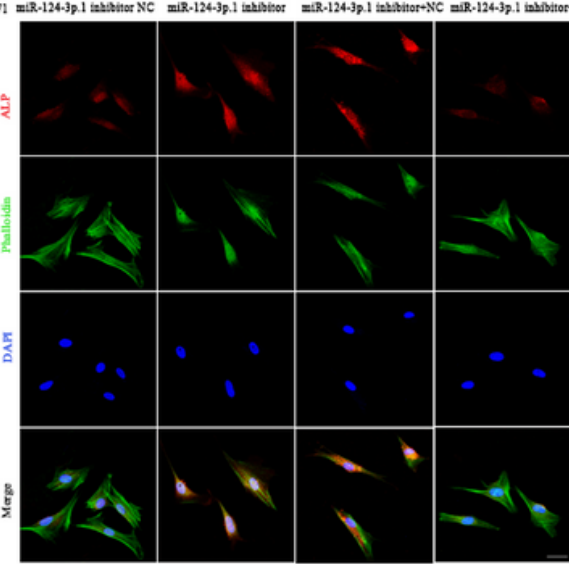

Figure 7 
Suppression of miR-124-3p.1 contributed to osteogenic differentiation of SCAPs via mediating MACF1 A. qRT-PCR analysis of MACF1 in miR-124-3p.1 inhibitor NC, miR-124-3p.1 inhibitor, NC/miR-124-3p.1 inhibitor and siMACF1/miR-124-3p. 1 inhibitor groups ( ${ }^{*} p<0.05$, $\left.{ }^{* *} p<0.01\right)$. B. Western blot analysis for MACF1 in miR-124-3p.1 inhibitor NC, miR-124-3p.1 inhibitor, NC/miR-124-3p.1 inhibitor and siMACF1/miR-124-3p.1 inhibitor groups. C. Relative quantitative analysis of western blot analysis for MACF1 ( $\left.{ }^{*} p<0.05, * \star p<0.01\right)$. D. Immunofluorescence analysis showed the expression of the protein expression of MACF1 (Scale bar= $50 \mu \mathrm{m}$ ). E. The mRNA levels of DSPP, RUNX2, ALP and OSX in miR-1243p.1 inhibitor NC, miR-124-3p.1 inhibitor, NC/miR-124-3p.1 inhibitor and siMACF1/miR-124-3p.1 inhibitor groups (Scale bar= $50 \mu \mathrm{m}$ ). F. Western blot analyses of DSPP, RUNX2, ALP and OSX in miR-124-3p.1 inhibitor NC, miR-124-3p. 1 inhibitor, NC/miR-124-3p. 1 inhibitor and siMACF1/miR-124-3p.1 inhibitor groups (Scale bar $=50 \mu \mathrm{m})$. G. Relative quantitative analysis of western blot analyses ( ${ }^{*}<0.05$, ** $p<0.01)$. H. Immunofluorescence staining of DSPP (Scale bar= $50 \mu \mathrm{m})$. I. Immunofluorescence staining of RUNX2 (Scale bar= $50 \mu \mathrm{m})$. J. Immunofluorescence staining of ALP (Scale bar= $50 \mu \mathrm{m})$. 


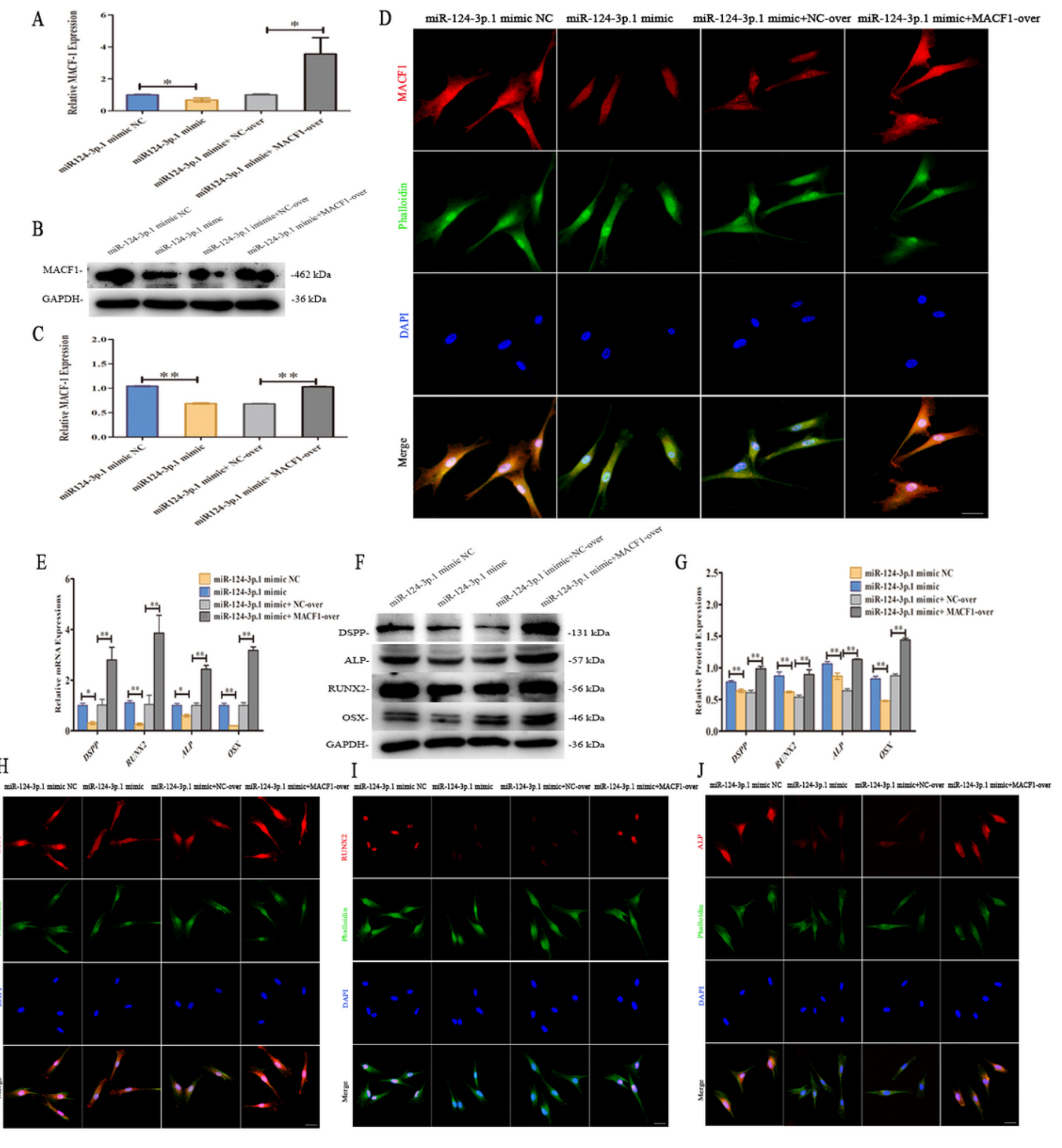

\section{Figure 8}

Overexpression of miR-124-3p.1 inhibited odontogenic and osteogenic differentiation via mediating MACF1 expression A. qRT-PCR analysis of MACF1 in miR-124-3p.1 mimic NC, miR-124-3p.1 mimic, NCover/miR-124-3p.1 mimic and MACF1-over/miR-124-3p.1 mimic groups ( ${ }^{*}<0.05$, ** $p<0.01$ ). B. Western blot analysis for MACF1 in miR-124-3p.1 mimic NC, miR-124-3p.1 mimic, NC-over/miR-124-3p.1 mimic and MACF1-over/miR-124-3p.1 mimic groups. C. Relative quantitative analysis of western blot analysis 
for MACF1 in $B(* p<0.05, * \star p<0.01)$. D. Immunofluorescence analysis showed the expression of the protein expression of MACF1 (Scale bar= $50 \mu \mathrm{m}$ ). E. The mRNA levels of DSPP, RUNX2, ALP and OSX in miR-124-3p.1 mimic NC, miR-124-3p.1 mimic, NC-over/miR-124-3p.1 mimic and MACF1-over/miR-124$3 p .1$ mimic groups (Scale bar $=50 \mu \mathrm{m}$ ). F. Western blot analyses of DSPP, RUNX2, ALP and OSX in miR124-3p. 1 mimic NC, miR-124-3p.1 mimic, NC-over/miR-124-3p.1 mimic and MACF1-over/miR-124-3p.1 mimic groups (Scale bar $=50 \mu \mathrm{m})$. G. Relative quantitative analysis of western blot analyses $\left({ }^{*} p<0.05, * \star\right.$ $p<0.01)$. H. Immunofluorescence staining of DSPP (Scale bar= $50 \mu \mathrm{m})$. I. Immunofluorescence staining of RUNX2 (Scale bar= $50 \mu \mathrm{m})$. J. Immunofluorescence staining of ALP (Scale bar= $50 \mu \mathrm{m})$. 
A
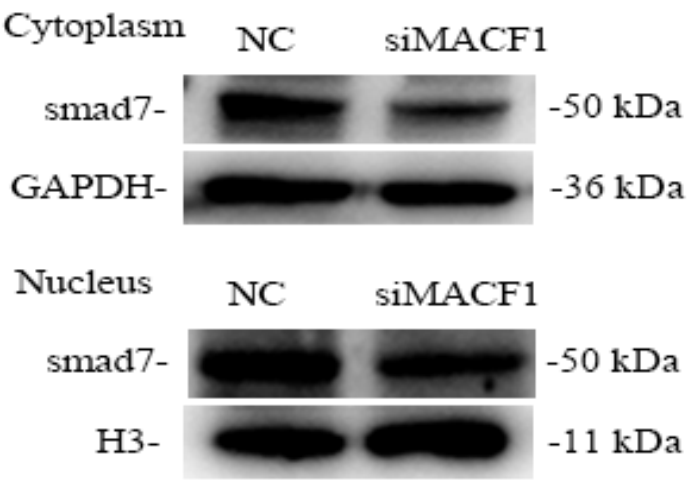

C

E

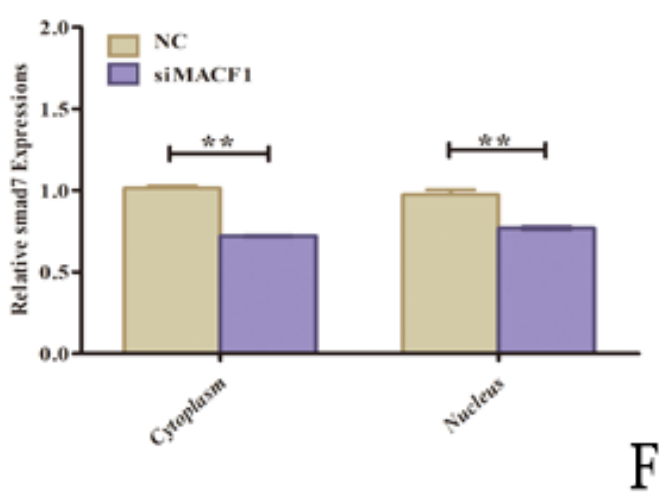

$\mathrm{F}$
B

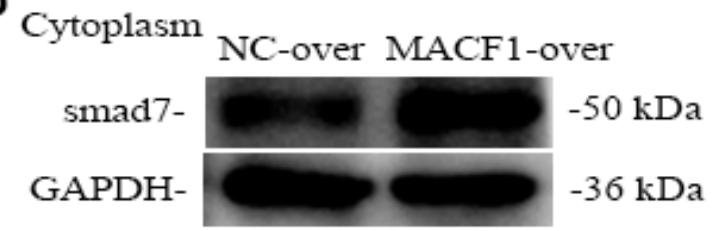

Nucleus NC-over MACF1-over

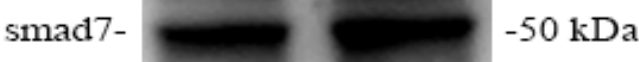

H3- $-11 \mathrm{kDa}$

D
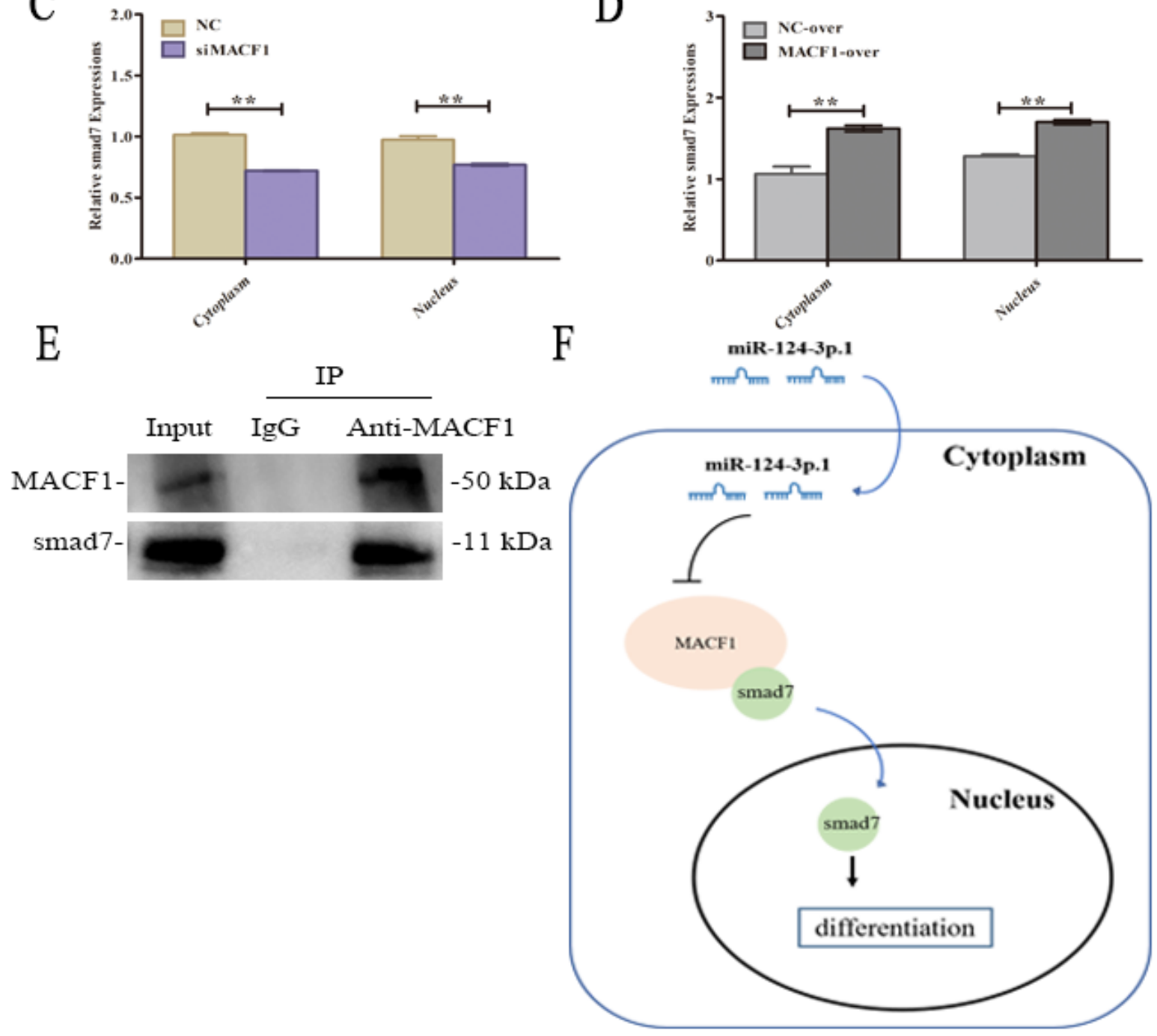

\section{Figure 9}

MACF1 interacts with smad7 in SCAPs A. Western blot analysis for smad7 in cytoplasm and nucleus in NC, siMACF1 groups. B, Western blot analysis for smad7 in cytoplasm and nucleus in NC-over, MACF1over groups. C. Relative quantitative analysis of western blot analysis for smad7 in $A$ ( ${ }^{*}<0.05$, ** $p<0.01)$. D. Relative quantitative analysis of western blot analysis for smad7 in $B(* p<0.05, * \star p<0.01)$. $E$. Co-IP assay showing interaction of MACF1 and smad7 in SCAPs. F. A mechanism diagram. 